\title{
On the dimensional characteristics and interpretation of vectors
}

\author{
Author: Gaurav Shantaram Biraris ${ }^{1}$ \\ Affiliation'1: KalpaSrusti, Pimpalner, Dist- Dhule, Maharashtra, India
}

\begin{abstract}
The paper proposes generalization of geometric notion of vectors concerning dimensionality of the configuration space. Trivial mapping between an algebraic vector space and Euclidean space is possible as the Euclidean space is able to configure all elements of the algebraic vector space. Such configuration relies on the notion of globally valid directions those satisfy the vector axioms upon their direct product with lengths. We prove that, certain type of ordered direction exists in each number of Euclidean dimensions along which elements of vector spaces can be interpreted. We show that such general ordered directions equivalently exist at each point in Euclidean space and there exists a special metric for each kind of the ordered direction. An algebraic structure of addition and scaling exists for the direct product of such directions and path lengths along such directions. The path length is in terms of the special metric that comes with each dimension. We further show that this consideration satisfies the vector axioms and leads to the complete normed space within the Euclidean space. A mathematical framework is built with 3 lemmas, 8 theorems and a conjecture. Application of the framework to locally $3+1$ dimensional universe leads to four fundamental versions as which a vector can exist geometrically. Thus any physical quantity in the universe should come in four versions of vectors as long as the underlying structure of spheres exists for the ordered directions.
\end{abstract}

Keywords: Vectors, Spheres, Banach spaces, Spacetime

MSCS (2010): 46B20, 51F99 


\section{Introduction}

The algebraic notion of vector as defined through the vector axioms provides abstract mathematical tool. On other hand, there is a valid geometric interpretation of the algebraic vectors as an arrow of certain length in its configuration space. The trivial configuration space for geometric interpretation of $\mathrm{n}$-dimensional vector is $\mathrm{n}$-dimensional Euclidean space. Possible geometric notion of the vector is just the direction along straight line. In this paper we present the possibility of the general geometric notion of vector by introducing a concept of ordered direction.

The algebraic vector space and corresponding configuration space are needed to be separately considered. By configuration space we mean the space wherein the elements of corresponding algebraic vector space are geometrically interpreted as the magnitudes along certain directions. For instance, the ordered n-tuple of numbers belonging to an algebraic space can be configured with numerical coordinates in $n$-dimensional space.

There is bijective relationship between an n-dimensional algebraic vector space and ndimensional Euclidean space, as the Euclidean space is able to configure all the elements of the algebraic vector space. In this regard, we find few geometrical characteristics of the Euclidean space associated with its dimensionality are of interest. Such dimensional characteristics provide ingredients for generalization of the direction as well as of magnitude, those are useful for establishing morphisms between algebraic vectors and the configured geometric vectors. Dimensional characteristics those come with every number of dimension are conventionally called as n-volume and n-plane. Both these characteristics imply classes of length, area, volume etc. and point, line, plane etc. respectively. There is another important dimensional characteristic: the class of distance, angle, solid angle etc. We find such characteristics useful for defining notion of vector. It will be proved that every number of Euclidean dimensions comes with a type of ordered direction and that of a measure, facilitating definition of corresponding dimensional vector. The theory model is developed in section 2, and case of the locally Minkowskian manifold as an example is concerned in section 3 .

In the paper, dimension is to be referred as Euclidean dimension. Also, $n$-dimensional space implies $n$-dimensional Euclidean space unless specified.

\section{Dimensional Characteristics}

An $m$-dimensional space that is embedded in $n$-dimensional space with $n>m$ leads to realization of certain geometric characteristic for each $m$. Examples are point, line, and plane for $m=0,1$, and 2 respectively. Let's denote the set of all points in $m$-dimensional space which may be embedded in higher spaces by $I_{m}$ i.e. $I_{m}=\left\{\left(x_{1}, x_{2}, \ldots, x_{m}\right) \mid x_{i} \in \mathbb{R}\right\}$. With this notation, points are identical with $I_{0} \mathrm{~s}$, lines with $\mathrm{I}_{1} \mathrm{~s}$ and planes are with $I_{2} \mathrm{~s}$. We will denote the highest dimensional space concerned for analysis (in which different $I_{m} \mathrm{~s}$ can be identified) by $X_{n}, n$ being the highest number of dimensions considered. For specific choice 
of $m$ except $m=n$, there are infinitely many $I_{m}$ s existing in $X_{n} . I_{m}$ s can be classified based on different values of $m$ i.e. $I_{m} \& I_{m}$, such that $m \neq m$ ' constitute to different types.

Lemma 1: $n+1$ types of $I_{\mathrm{m}}$ s exist in $n$-dimensional space.

Proof: At most $n$ number of mutually perpendicular lines can be drawn at a point in $X_{n}$. A subspace of $X_{n}$ consisting of the $m$ mutually perpendicular lines is nothing but $I_{m}$. Hence $I_{m}$ such that $0<m \ngtr n$ can be manifested at each point in $X_{n}$. In this way, one can manifest $n$ types of $I_{m}$ s differing by number of dimensions. Additionally the points exist as $I_{0}$ s; thus in total $n+1$ types of $I_{m}$ s exist in $X_{n}$.

Quantification of subsets of the configuration space is essential for the analysis. Quantification of subsets of the $I_{m}$ s would provide a useful tool for quantitative analysis. Any quantification in $X_{n}$ is possible through quantifications of subsets of various $I_{m}$ s only. We can quantify subsets of $I_{m} \mathrm{~s}$ by defining appropriate measures on them. Lebesgue measure provides trivial quantification of subsets of the $I_{m} \mathrm{~s}$.

Let's denote the quantification of a proper subset $\mathrm{E}$ of $I_{m}$ given by Lebesgue measure on it by $L_{m}(\mathrm{E})$. For general expression, we can omit the $\mathrm{E}$ in bracket as long as possible. Thus length, area and volume are $L_{1}, L_{2}$ and $L_{3}$ respectively. $I_{0}$ s being just the points, don't have any proper subset. Hence we can't define the Lebesgue measure on $I_{0}$; hence there is no existence of $L_{0}$.

Going a step forward with the lemma 1, an $n$-dimensional geometrical object i.e. proper subset of $X_{n}$ will have $n$ types of $L_{m}$ s obtained by Lebesgue measures on all the types of corresponding $I_{m}$ s (enclosed by boundary of the object) except on $I_{0}$. For instance, a 3dimensional object has length (or perimeter), area (or surface area) and volume. We can regard the $L_{m}$ s as trivial geometrical properties (or quantifications); in $X_{n}$ any subset would have at most $n$ types of geometrical properties.

Definition 1: In $X_{n} n \geq m, m+1$ points as relative position of one point with respect to the remaining $m$ points can be specified by single real valued function defined as m-dimensional Geometrical Relation $\left(R_{m}\right)$ of the point with respect to the $m$ points. i.e. $R_{m}: X_{n} \rightarrow \mathbb{R}$

Such functions do exist in Euclidean geometry; we can check that distance and angle are the functions which fit in above definition.

Distance is $R_{1}$ which specifies positions of two points i.e. relative position of one point with respect to another point. Angle is $R_{2}$ obtained by relative positions of three pointsas of one points with respect to two points. In similar fashion, solid angle is $R_{3}$ obtained from four points (relative positions of a point with respect to three points). Inversely, the angle and solid angle yield the set of points on sphere of certain radius. In general, they lead to degeneracy of the points along same radius ray for different concentric spheres. 
Distance, angle and solid angle can be defined by using concept of the spheres. Hence spheres seem to be useful for defining $R_{m}$ s. Topology can be induced on $X_{n}$ by considering collection of all the open subsets of $X_{n}$. Spheres exist in general topological space. Let's denote an $m$-sphere in $X_{n}$ by $S_{m}$ i.e. $S_{m} \equiv\left\{x \in \mathbb{R}^{m}:\|x\|=\mathrm{r}\right\}$. By a sphere about a point we will mean the sphere having centre at the point.

$R_{m}$ s would be useful for dynamical analysis in $X_{n}$ as they facilitate specification of relative positions of points. Here we conjecture,

Conjecture: The $m$-dimensional geometrical relation $\left(R_{m}\right)$ of $m$ points with respect to a point $x$ is given by

$$
R_{m}=\frac{L_{m-1}(E)}{\mathrm{r}^{\mathrm{m}-1}}
$$

Where, $E$ is the $m$ vertex open set formed by projections of the $m$ points on a $S_{m-1}$ having centre at $x$. And $r$ is the radius of the $S_{m-1}$ on which $E$ is measured.

$m$-dimensional geometrical relation of an open set $(E)$ formed by the $m$ points of interest on the sphere can be written as: $R_{m}(E)$ with respect to centre point of the sphere. For consideration of $E$ and quantification $R_{m}$, a frame in $X_{n}$ is essential. The frame should facilitate the $S_{m-1}$ with the implied point at its centre.

Lemma 2: $R_{m}$ defined by the conjecture is a measure in $\mathrm{X}_{\mathrm{n}}$

Proof: In $X_{n} n \geq m$, embedding of $S_{m-1}$ is possible. Hence there exists $S_{m-l}$ about each point. Further, any point can be projected on a $S_{m-l}$ along the radial direction.

Thus any $m$ points can be projected on a $S_{m-l}$, so that on the spherical surface, they lead to an open set $E$ (analogous curved polygon) fixed by the projections as vertices. Let $\sum$ be a $\sigma$-ring of open sets over the $S_{m-1}$; then the $R_{m}$ given by (1) is a function from $\sum$ to $\mathbb{R}$. $L_{m-1}$ of any $E$ is non-negative and therefore $R_{m}$ is non-negative as $\mathrm{r}$ too is non-negative. i.e for all sets $E$ on any $S_{m-1}$,

$$
R_{m}(E) \geq 0
$$

As we are considering open sets $E$, an empty set would be that which contain no point. For the empty set $\varnothing$ containing no points, $L_{m-l}(\varnothing)=0$; thus by (1),

$$
\text { i.e. } R_{m}(\varnothing)=0
$$

For all countable collections $\left\{E_{i}\right\}_{i \in N}$ of pairwise disjoint sets in $\Sigma$, by the conjecture:

$$
\sum_{i=1} R_{m}\left(E_{i}\right)=\sum_{i=1} \frac{L_{m-1}\left(E_{i}\right)}{\mathrm{r}^{\mathrm{m}-1}}
$$


As the sets in $\left\{E_{i}\right\}_{i \in N}$ are disjoint and $L_{m-1}$ is a measure, $\sum_{i=1} \frac{L_{m-1}\left(E_{i}\right)}{\mathrm{r}^{\mathrm{m}-1}}=\frac{L_{m-1}\left(\bigcup_{i=1} E_{i}\right)}{\mathrm{r}^{\mathrm{m}-1}}$

Hence rewriting the RHS by using the conjecture,

$$
\sum_{\mathrm{i}=1} R_{m}\left(E_{i}\right)=R_{m}\left(\bigcup_{i=1} E_{i}\right)
$$

Essential conditions for a function to be measure are non-negativity, null empty set and countable additivity (or $\sigma$-additivity) which are proved by (2), (3) and (4) respectively. Hence the $R_{m}$ is a measure on $S_{m-1}$ embedded in $X_{n}$.

$S_{n-1}$ about any point exists in $X_{n} . R_{m}$ is defined for $m$ points with respect to the centre point (the centre point can be fixed by a specific frame). Any $m$ points in $X_{n}$ can be radially projected on a $S_{m-1} . S_{m-1}$ is subset of same centered $S_{n-1}$ of same radius; hence any $S_{m-l}$ needed to realize radial projections of the $m$ points exists on the $S_{n-1}$. Thus $R_{m}$ can be used for any $m+1$ points in $X_{n}$ by proper choice of the $S_{m-1}$ in certain frame; hence it is measure in whole $X_{n}$.

For every value of an $R_{m}$, because of continuity of $S_{m-1}$ and $L_{m-1}$, we can find at least one corresponding point in $X_{n}$ in fixed frame of $m$ points. Hence $R_{m}$ is surjective map from $S_{m-1}$ to real numbers, $R_{m}: S_{m-1} \rightarrow \mathbb{R}$.

For $m=1$, the conjecture is meaningless due to geometry of $S_{0}$. The end points of a diameter (arbitrary line segment) represent $S_{0}$; but there is no existence of proper subsets of $S_{0}$. This makes $L_{m-l}(E)$ in (1) meaningless. Hence the conjecture is meaningless for $m=1$. However, we can identify $R_{1}$ by using $S_{0}$ and obeying definition 1 . The $R_{1}$ should be able to specify relative positions of two points. Any two points can be considered to lie on a corresponding $S_{0}$. A $S_{0}$ lies on a line i.e. $I_{1}$. Thus the Lebesgue measure on subsets of the line can be used as $R_{1}$. That is, distance can be identified as the $R_{1}$. Such $R_{1}$ too is a measure in $X_{n}$. Though the conjecture is meaningless for $m=1$, we can specially consider $R_{1}$ to be distance between two points as long as it doesn't make contradiction with the framework.

$R_{m}$ and $L_{m}$ both are measures in $X_{n} . L_{m}$ is measure of proper subsets of $I_{m}$, and $R_{m}$ is measure of relative positions of points with respect to a point in $I_{m}$. For a dynamical analysis where changes happen with time, essential characteristic of a measure to be parameter is that continuous variation in its real value should be possible in certain reference frame. Existence of Cauchy sequences with real number images is essential for this. $R_{m}$ is better measure for studying dynamics where out of $m+1$ points, $m$ can be fixed as the references frame and 
variation in positions of the remaining point object can be analyzed as variation in its $R_{m}$ in the frame.

As $n$ types of spheres exist in $X_{n}$, the $n$ types of $R_{m}$ s such that $1 \leq m \leq n$ do exist. Variation in position of a point object with respect to certain reference frame can be measured in form of its varying $R_{m} \mathrm{~s}$. Thus in $n$-dimensional space, a motion can be characterized by any of $n$ types of $R_{m}$ s as suitable. In 3-dimensional space, a motion can be described in terms of variation in distance or that in angle or even in solid angle whichever is suitable. We can make difference between general direction and ordered direction. Direction is the manifestation of variation in positions of a point object in its neighborhood in a reference frame. It can be configured by variation of $R_{m} \mathrm{~s}$ in the frame. An ordered direction is special in a sense that it is realized in ordered pattern and can be parameterised by single type of $R_{m}$.

Definition 2: A continuous path $\Upsilon$ in a neighborhood of point $x$ in $X_{n}$ is defined as an m-dimensional ordered direction $\left(\mathscr{D}_{\mathrm{m}}\right)$ if there exists a bijection $R_{m}: \Upsilon \rightarrow \mathbb{R}$ for every point $x_{\Upsilon} \in \Upsilon$ in a frame.

When all points on a path are described by values of single typed geometrical relation in a frame, then the direction described by the path is to be called as ordered direction. Rectilinear path is set of points that can be analyzed by concerning only distances in certain frame. Curvilinear path is set of points that can be analyzed by concerning distances and angles in a frame. For a random path, there is no frame wherein all the points can be described by angles or distances only. On other hand, angular path can be described by concerning only angles in a frame. Thus rectilinear and angular are ordered directions, while curvilinear and the random aren't. It is easy to identify rectilinear direction as $\mathfrak{D}_{1}$ (as we have identified $R_{1}$ with distance) and angular direction as $\mathfrak{D}_{2}$. Such directions are globally valid throughout the path. They are not local directions defined by the tangents to path.

The higher $R_{m}$ s are periodic functions, thus they are surjective maps. Yet they offer bijection when considered within single period i.e. as long as the path $\Upsilon$ in a neighborhood of $x$ doesn't form a loop. Definition 2 defines the ordered directions in neighbourhood of a point. Thus, the bijection in the neighborhood suffices here.

Lemma 3: The set of points along a $\mathfrak{D}_{m}$ forms metric space with a special metric, and Cauchy sequences along $\mathfrak{D}_{\mathrm{m}}$ exist for every point along the $\mathfrak{D}_{\mathrm{m}}$.

Proof: By definition 2, all the points along $\mathscr{D}_{\mathrm{m}}$ can be mapped with different real numbers by $R_{m}$ in certain frame. The conjecture implies that all these points lie on a $S_{m-1}$. To map each point $x$ along the $\mathscr{D}_{\mathrm{m}}$, corresponding set $E_{x}$ is needed to be realized on the $S_{m-1}$. For this, the frame defined by $m$ fixed points as $m-1$ points on the $S_{m-1}$ and remaining one at the centre of the $S_{m-1}$ can be used. In this frame, the open set formed on the $S_{m-1}$ by the $m-1$ reference points and $x$ is the $E_{x}$. This $E_{x}$ gives $R_{m}(x)$ by (1). In this way we can get the real number $R_{m}(x)$ in the frame for each point $x$ along the $\mathscr{D}_{\mathrm{m}}$. 
Now in same frame, for $x, y$ on the $\mathfrak{D}_{m}$, let

$$
d(x, y)=\left|R_{m}(x)-R_{m}(y)\right|
$$

Then, $d(x, y)=d(y, x)$. Also, $d(x, y) \geq 0$ for any $x$ and $y$. Further, $d(x, y)=0$ implies $R_{m}(x)=R_{m}(y)$. But as per definition $2, R_{m}$ on the $\mathscr{D}_{\mathrm{m}}$ is a bijection in neighbourhood of $x$ and $y$. Hence $R_{m}(x)=R_{m}(y)$ implies $x=y$. That is, $d(x, y)=0$ implies $x=y$.

Now consider $d(x, z) \leq d(x, y)+d(y, z)$. It's true if two of $x, y, z$ are same or equivalently: any of $R_{m}(x), R_{m}(y), R_{m}(z)$ are equal. Let's assume $R_{m}(x)<R_{m}(z)$. Then there are 3 possibilities as $R_{m}(y)<R_{m}(x)<R_{m}(z), R_{m}(x)<R_{m}(y)<R_{m}(z), R_{m}(x)<R_{m}(z)<R_{m}(y)$. In the first case $d(x$, $z)<d(y, z)$ and in the third case $d(x, z)<d(x, y)$, so in both these cases we get the strict inequality $d(x, z)<d(x, y)+d(y, z)$. In the second case we get the equality: $d(x, z)=d(x, y)+$ $d(y, z)$. The case of $R_{m}(x)>R_{m}(z)$ yields the inequality in similar way. This proves the triangle inequality.

This makes it clear that $d(x, y)$ for the points along a $\mathscr{D}_{\mathrm{m}}$ bears symmetry, nonnegetivity, identity of indiscernibles, and triangle inequality. Hence set of the points along a $\mathfrak{D}_{\mathrm{m}}$ form a metric space with the special metric defined in (5).

Furthermore, consider a sequence of points $\left\{x_{i}\right\}=x_{1}, x_{2}, x_{3}, \ldots$ along a $\mathfrak{D}_{\mathrm{m}}$ in $X_{n}$. Then the sequence $\left\{x_{i}\right\}$ is identified by varying values of $R_{m}$ in a constant frame. The points are identified by values of $R_{m}$ in the frame i.e. $x_{i}=\frac{L_{m-1}\left(E_{i}\right)}{\mathrm{r}^{m-1}}$ where, $E_{i}$ is the set defined by the point $x_{i}$ and the reference points on the $S_{m-1}$ of the frame. As the $m$ points are fixed due to frame, only $x_{i}$ determines $E_{i}$. As range of $L_{m-1}\left(E_{i}\right)$ is $\mathbb{R}$, for any positive real number $\varepsilon$ and $\mathrm{N}<$ i, j, $N \in \mathbb{N}$ we can obtain $\left|L_{m-1}\left(E_{i}\right)-L_{m-1}\left(E_{j}\right)\right| \leq \varepsilon$. This ensures existence of the Cauchy sequence $\left\{L_{m-1}\left(E_{i}\right)\right\}$. And as $R_{m}$ is division of $L_{m-1}\left(E_{i}\right)$ by just a positive number $\mathrm{r}^{m-1}$, for any positive real number $\varepsilon$ and $\mathrm{N}<\mathrm{i}, \mathrm{j}, \mathrm{N} \in \mathbb{N}$ we have $\left|\frac{L_{m-l}\left(E_{i}\right)}{\mathrm{r}^{\mathrm{m}-1}}-\frac{L_{m-l}\left(E_{j}\right)}{\mathrm{r}^{\mathrm{m}-1}}\right| \leq \varepsilon$, equivalently we have $\left|R_{m}\left(x_{i}\right)-R_{m}\left(x_{j}\right)\right| \leq \varepsilon$. This proves that the Cauchy sequence along a $\mathfrak{D}_{\mathrm{m}}$ exists.

As all the points along a $\mathscr{D}_{\mathrm{m}}$ are described by single type of geometrical relation i.e. $R_{m}$, such direction can be parameterized by the $R_{m}$ in the frame. As Cauchy sequences for the $R_{m}$ s exist, their continuous variations are possible.

If a point object is taking different positions $x_{\Upsilon} \in \Upsilon$ varying with time, then the path $\Upsilon$ describes the motion. Thus the motions of point objects along the $\mathfrak{D}_{\mathrm{m}} \mathrm{s}$ can simply be defined as ordered motions. Then according to definition 2 , an observer in $n$-dimensional space can manifest $m$-dimensional ordered motions such that $1 \leq m \leq n$. Hence in $n$-dimensional space, one can manifest at most $n$ types of ordered motions (and directions). Thus in 3-dimensions, 
one can manifest 3 types viz. rectilinear $\left(\mathfrak{D}_{1}\right)$, angular $\left(\mathfrak{D}_{2}\right)$ and solid angular $\left(\mathfrak{D}_{3}\right)$ of ordered motions.

Vector has magnitude and direction. We can generalize the notion of vector while preserving the algebraic properties. The directions $\mathscr{D}_{\mathrm{ms}}$ would be useful for interpreting/identifying algebraic vectors in $X_{n}$.

Theorem 1: In a frame in $X_{n}$, continuous variation in $R_{m} m \leq n$ indicates direction along the. $I_{m}$.

Proof: In a frame in $X_{n}$ the $R_{m}$ is a map from a $S_{m-1}$, defined for all points on the $S_{m-1}$.

$R_{m}=\frac{L_{m-1}(E)}{\mathrm{r}^{\mathrm{m}-1}} \quad E$ is the set formed by the $m$ points on the $\mathrm{S}_{\mathrm{m}-1}$ in the frame. $L_{m-1}\left(E_{i}\right)$ is conventionally called as ( $m-1)$-surface area. It for entire $S_{m-1}$ is given [1] as

$$
L_{m-1}\left(S_{m-1}\right)=\frac{2 \pi^{\mathrm{m} / 2}}{\Gamma\left(\frac{\mathrm{m}}{2}\right)} \mathrm{r}^{\mathrm{m}-1}
$$

where, $\Gamma$ denotes the gamma function. For a set $E_{i}$ formed on the sphere, the $L_{m-1}\left(E_{i}\right)$ will be fraction of (6).

$$
\text { i.e. } \quad L_{m-l}\left(E_{i}\right)=\frac{2 \mathrm{f}_{\mathrm{i}} \cdot \pi^{\mathrm{m} / 2}}{\Gamma\left(\frac{\mathrm{m}}{2}\right)} \mathrm{r}^{\mathrm{m}-1}, 0 \leq \mathrm{f}_{\mathrm{i}} \leq 1
$$

putting this in the conjecture (1) we get $R_{m}=\frac{2 \mathrm{f}_{\mathrm{i}} \cdot \pi^{\mathrm{m} / 2}}{\Gamma\left(\frac{\mathrm{m}}{2}\right)}$

This new expression (7) of the $R_{m}$ indicates that in a frame, $R_{m}$ is defined irrespective of radius of the sphere. As $S_{m-1}$ exists in $m$-dimensional space (and concentric $S_{m-1}$ s cover the $m$-dimensional space), now $R_{m}$ can be thought as a function on whole $m$-dimensional space spanned by the $S_{m-1}$ s. But $m$-dimensional space embedded in higher dimensional space is nothing but an $I_{m}$. Thus $R_{m}$ is a morphism from the $I_{m}$ to $\mathbb{R}$. This would be a surjection from $I_{m}$ to $\mathbb{R}$.

Thus any point $x$ in an $I_{m}$ can be identified by a value of $R_{m}$ as the $R_{m}(x)$ in the frame. Due to existence of Cauchy sequence for $R_{m}$ in a neighborhood of $x$ on $S_{m-1}$ of certain radius, the $R_{m}$ will either increase or decrease or can be unchanged for each neighboring point. We can assign directions to such variations, suppose we assign direction $\mathscr{D}_{\mathrm{m}}$ to manifestation of increasing $R_{m}$, then $-\mathscr{D}_{\mathrm{m}}$ will be manifestation of decreasing $R_{m}$. No change in the $R_{m}$ of neighboring point will not lead to manifestation of the direction $\mathfrak{D}_{\mathrm{m}}$ as on the ordered path $R_{m}$ 
is bijection according to definition 2. Conclusively, any change in $R_{m}$ manifests single direction $\mathscr{D}_{\mathrm{m}}$ in (or along) the $I_{m}$. And, no change in $R_{m}$ manifests no $\mathscr{D}_{\mathrm{m}}$.

$\mathfrak{D}_{\mathrm{m}}$ is actually an algebraic notion for the direction realized by varying $R_{m}$. For manifestation of direction along the $I_{m}$, there should be continuous variation in $R_{m}$ so that $\mathscr{D}_{\mathrm{m}}$ is continuously manifested. If value of $R_{m}$ for neighbouring points remains same, then no $\mathfrak{D}_{\mathrm{m}}$ is realized.

$I_{m}$ is collection of points that is equivalent to $m$-dimensional space. Thus coordinate chart on an $I_{m}$ is possible by identifying points in $I_{m}$ with elements of $\mathbb{R}^{\mathrm{m}}$ as $\mathrm{C}: I_{m} \rightarrow \mathbb{R}^{\mathrm{m}}$. But Theorem 1 and eq. (7) suggest that the points in $I_{m}$ can be identified by $R_{m}$ with elements of $\mathbb{R}$ i.e. $\mathrm{C}_{\mathrm{m}} \equiv R_{m}: I_{m} \rightarrow \mathbb{R}$. Thus $R_{m}$ may be thought as the 1-dimensional co-ordinate system for $m$-dimensional space; but it has non empty kernel, at least all points along a radius of the $S_{m-1}$ are mapped to same element of $\mathbb{R}$. Further, all the points having same $R_{m}$ (those don't manifesting the $\mathscr{D}_{\mathrm{m}}$ ) are too mapped to same element of $\mathbb{R}$. However, we get a useful corollary from theorem 1 .

Corollary 1.1: Any m-dimensional space can be identified with set of real numbers by $R_{m}$ as the chart $\mathrm{C}_{\mathrm{m}} \equiv R_{m}: I_{m} \rightarrow \mathbb{R}$ in certain frame.

The geometrical relations provide trivial real numbered chart for corresponding dimensional space.

All points in neighbourhood of a point $x \in \mathrm{X}_{\mathrm{n}}$ having same $R_{m} \mathrm{~s}$ in the frame constitute to kernel of the chart $\mathrm{C}_{\mathrm{m}}$. As $R_{m}$ is same for all points along a radial direction, it is inevitably non-injective surjective map. A good coordinate chart is needed to be injective and surjective. In order to achieve this, extra components should be considered in the chart amenable to distinguish the kernel points. This can be done by considering extra components from lower dimensional geometrical relations i.e. $R_{m}$ 's such that $m^{\prime}<m$ in the chart. For example, points along same radial direction in the frame having same $R_{m}$ can be distinguished by considering the radial distance (i.e. $R_{1}$ ) as an additional component of the chart. Two points having same $R_{m}$ in a frame can be distinguished by values of $R_{m}$, in a subframe. By subframe we mean subset of the frame amenable to provide $m$ ' fixed reference points in order to quantify $R_{m}$, of a point. By adopting lower dimensional geometrical relations in the chart in order to make it bijective, we would be needed to consider all the $m$ types of $R_{m} m=1,2,3 . . m$. Thus eventually we get map of $I_{m}$ to $\mathbb{R}^{\mathrm{m}}$. In other words, set of all kinds of the geometrical relations would provide a potential co-ordinate chart for $\mathrm{C}: I_{m} \rightarrow \mathbb{R}^{\mathrm{m}}$.

Realization of a path in a neighborhood can be interpreted as the direction defined by the path. Before exploring characteristics of the directions, let's make two definitions. 
Definition 3: A set of directions $\mathbf{S}=\left\{D_{i}\right\}$ near a point is to be called as mutually exclusive directions if realization of a direction $D_{j} \in \mathbf{S}$ along a path in $X_{n}$ implies no realization of all other directions $D_{i \neq j} \in \mathbf{S}$ along same path.

Definition 4: A set of directions $\mathbf{S}=\left\{D_{i}\right\}$ near a point is to be called as collectively exhaustive directions if no direction other than elements of $\mathbf{S}$ can be realized along any path in neighborhood of any point in $X_{n}$.

Definitions of mutually exclusive and collectively exhaustive directions can be used for ordered directions. This is because the ordered directions are special type (subsets with respect to underlying paths) of general directions.

Theorem 2: In n-dimensional space, continuous variation in position of a point object can lead to manifestation of $n$ types of mutually exclusive ordered directions.

Proof: In an $n$-dimensional space $X_{n}, S_{m-1}$ such that $m$ being at most $n$ exists. Thus highest dimensional spherical path would exist on $S_{n-1}$. The direction along $S_{n-1}$ configured by continuously varying $R_{n}$ in a frame is $\mathscr{D}_{\mathrm{n}}$. As implied by definition $2, \mathfrak{D}_{\mathrm{n}}$ isn't manifested on the continuous path defined by the non varying $R_{n}$ value because of conditional bijection of $R_{n}$ in definition of $\mathscr{D}_{\mathrm{n}}$.

If in neighborhood $N_{x}$ of a point $x$ in $X_{n}, R_{n}$ values of all the points in a frame are same, then $N_{x}$ constitutes kernel of the direction implied by $R_{n}$. The $n$ reference points being constant in the frame, the set $E$ is identified by point $x$ only. Hence it is fair to call the $L_{m}(E)$ be $L_{m}$ of $x$ i.e. Lebesgue measure of the point. From (1) we infer that same $R_{n}$ implies same $L_{n-1}$ of the points in the frame. If a Lebesgue measure of continuous (neighbouring) points is same, then we can find a subframe wherein an ordinate (in same dimension) of all the points is same. That is- all those points lie in a lesser dimensional cross section of the space. The cross sectional space accommodating all those point has number of dimensions one lesser than that of the prior space. In short, if $L_{m}$ of continuous points is same, then all those points lie in single $I_{m-1}$ (i.e. a lesser dimensional section of the $I_{m}$ ). Thus the points in $N_{x}$ having same $R_{n}$ should lie on cross section of the $S_{n-1}$ with the $I_{n-1}$ containing $N_{x}$. Cross section of the $S_{n-1}$ with $I_{n-1}$ is nothing but the $S_{n-2}$. Hence $N_{x}$ lies on a $S_{n-2}$ which is subset of $S_{n-1}$. Frames for $S_{n-2}$ are subsets of frames for $S_{n-1}$; thus in the same frame we can obtain the map $R_{\mathrm{n}-1}: N_{x} \rightarrow \mathbb{R}$ for the points which do not lead to manifestation of $\mathscr{D}_{\mathrm{n}}$. Continuous varying $R_{n-1}$ implies direction $\mathscr{D}_{\mathrm{n}-1}$ along the $S_{n-2}$. The general $R_{m} \mathrm{~s}$ aren't injective (and bijective) but the $\mathscr{D}_{\mathrm{m}}$ are defined by the bijection i.e. $\mathscr{D}_{\mathrm{m}} \mathrm{s}$ pick up the subsets on which corresponding $R_{m} \mathrm{~s}$ are bijective. Hence on the $S_{n-2}$ (equivalently in $N_{x}$ ), there will be some continuous points (let's identify their set be $N_{x}$ ') leading to a path for which $R_{n-1}$ is constant and not manifesting of $\mathscr{D}_{\mathrm{n}-1}$. This is possible only when $N_{x}{ }^{\prime} \in S_{\mathrm{n}-3} \subset S_{\mathrm{n}-2}$. Then paths on the $S_{n-3}$ for which $R_{n-2}$ uniquely identifies the points, are manifested as $\mathscr{D}_{\mathrm{n}-2}$. But yet there would be continuous points having same $R_{n-2}$. Such points must lie on $S_{n-4}$ leading to $\mathfrak{D}_{\mathrm{n}-3}$. Following this scheme, on the most general sphere i.e. $S_{n-1}$, different ordered directions are manifested as $\mathscr{D}_{\mathrm{n}}, \mathfrak{D}_{\mathrm{n}-1}$, 
$\mathscr{D}_{\text {n-2 }}, \ldots, \mathfrak{D}_{3}, \mathscr{D}_{2}$. Direction $\mathfrak{D}_{2}$ is manifested on $S_{1}$, and on $S_{1}$, there are no two points having same $R_{2}$ i.e. angle in a frame.

In addition to these ordered directions, a type of ordered directions is possible along the paths that change radius of the spheres considered so far. This is manifestation of direction along a straight line $\ell$, in terms of distances as $\mathrm{R}_{1}: \ell \rightarrow \mathbb{R}$. Straight line is nothing but $I_{1}$. Such rectilinear path is manifested as primary ordered direction $\mathfrak{D}_{1}$. Hence there are $n$ types of ordered directions $\mathscr{D}_{\mathrm{i}}, 1 \leq \mathrm{i} \leq \mathrm{n}, \mathrm{i} \in \mathbb{N}$ in $X_{n}$.

When $R_{m}$ doesn't lead to identification of difference in points along a path, then we adopt $R_{m-1}$ to identify the points. Equivalently when $\mathscr{D}_{\mathrm{m}}$ is not manifested along a path, then $\mathscr{D}_{\mathrm{m}-1}$ can be manifested; and sequentially when $\mathfrak{D}_{\mathrm{m}-1}$ isn't manifested, we may manifest $\mathfrak{D}_{\mathrm{m}-2}$ by employing $R_{m-2}$. This sequence is followed till manifestation of $\mathfrak{D}_{1}$. Further, any two neighboring points having varying $R_{m}$ don't lie on same $S_{m-2}$ (or lower spheres), thus they can't be distinguished by $R_{m-1}$ (or lower dimensional geometrical relations). That is when $\mathfrak{D}_{\mathrm{m}}$ is manifested, then no lower dimensional ordered direction is manifested. Hence no two ordered directions $\mathscr{D}_{\mathrm{i}}$ are manifested on same path in the frame. In other words, the $n$ types of ordered directions $\mathscr{D}_{\text {is }}$ realized in $X_{n}$ are mutually exclusive.

In $X_{n}$, there exist infinitely many $\mathscr{D}_{\mathrm{m}}$ s for any $m<n$. This is because with this condition, infinitely many $S_{m-1}$ s exist about a point in $X_{n}$. While there only one $S_{n-1}$ exists at a point; thus single $\mathscr{D}_{\mathrm{n}}$ is manifested. This is a useful corollary.

Corollary 2.1: In $X_{n}$ there exists infinitely many $\mathscr{D}_{\mathrm{ms}}$ for specific $m 1 \leq \mathrm{m}<\mathrm{n}, \mathrm{m} \in \mathbb{N}$, but only one $\mathfrak{D}_{\mathrm{n}}$.

Ordered directions are manifested by paths on spheres or along straight lines. But there are general paths which are neither along any sphere nor along lines. Such paths manifest directions different from ordered directions. Therefore different directions can be manifested in $X_{n}$ which aren't ordered direction. This leads to following proposition.

Corollary 2.2: The $n$ types of ordered directions manifested in $X_{n}$ aren't collectively exhaustive.

The corollary 2.1 states existence of infinitely many $\mathfrak{D}_{\mathrm{mS}}$ for certain $m$ in higher dimensional space. Existence of infinitely many $S_{m-1}$ s having same centre is the reason behind this proposition. The ordered direction along each $S_{m-1}$ is regarded as different from those along others while all of them belonging to same class due to dimensionality $\mathrm{m}$. For analytical purpose, relationship between various $\mathscr{D}_{\mathrm{ms}}$ is essential. For now, we can make two definitions which would help for establishing relationship between different $\mathfrak{D}_{\mathrm{m}}$. 
Definition 5: An m-dimensional ordered direction $\mathfrak{D}_{\mathrm{m}}{ }^{\mathrm{a}}$ is defined to be dependent on another m-dimensional ordered direction $\mathfrak{D}_{\mathrm{m}}{ }^{\mathrm{b}}$ if projection of any path along $\mathfrak{D}_{\mathrm{m}}{ }^{\mathrm{a}}$ gives certain path on $\mathfrak{D}_{\mathrm{m}}{ }^{\mathrm{a}}$.

Definition 6: An m-dimensional ordered direction $\mathfrak{D}_{\mathrm{m}}{ }^{\mathrm{a}}$ is defined to be independent of another m-dimensional ordered direction $\mathfrak{D}_{\mathrm{m}}{ }^{\mathrm{b}}$ if projection of any path along $\mathfrak{D}_{\mathrm{m}}{ }^{\mathrm{a}}$ gives no path on $\mathfrak{D}_{\mathrm{m}}{ }^{\mathrm{a}}$.

Projection of a path along $\mathscr{D}_{\mathrm{m}}{ }^{\mathrm{a}}$ on $\mathfrak{D}_{\mathrm{m}}{ }^{\mathrm{b}}$ is characterized by extremities of the path. The projection is realised by the right angle at the intersection with target entity. If projections of both the path extremities lie on different points along $\mathcal{D}_{\mathrm{m}}{ }^{\mathrm{b}}$ then the certain path length of projection is realized. If both the projections lie at same point along $\mathcal{D}_{\mathrm{m}}{ }^{\mathrm{b}}$ then no path on $\mathfrak{D}_{\mathrm{m}}{ }^{\mathrm{b}}$ is realized. A rough idea of above definitions is that the Perpendiculal directions are independent of each other, while inclined directions have some interdependency.

Path length along $\mathscr{D}_{\mathrm{ms}}$ can be measured in terms of the metric induced by $R_{m}$. Independent $\mathscr{D}_{\mathrm{m}}$ have projection of zero path length on each other, as there is absence of the projected path. When two $\mathscr{D}_{\mathrm{m}} \mathrm{s}$ are dependent on each other, projections of the path lengths along them on each other will differ from the original path lengths. More or less difference in the magnitude of projection should imply more or less dependency respectively. But we get a special case where path length of the projection is exactly equal to original path length. This case can be used for defining equivalence between both the $\mathscr{D}_{\mathrm{m}} \mathrm{s}$, where the path lengths along $\mathscr{D}_{\mathrm{m}} \mathrm{s}$ are completely depend on each other. By definition 2 , each point along a $\mathfrak{D}_{\mathrm{m}}$ is determined by $R_{m}$. Thus equal path lengths in terms of $R_{m}$ imply equivalence of all the points along the paths.

Definition 7: Two m-dimensional ordered directions $\mathscr{D}_{\mathrm{m}}{ }^{\mathrm{a}}$ and $\mathfrak{D}_{\mathrm{m}}{ }^{\mathrm{b}}$ are defined to be equivalent if projection of any path along $\mathfrak{D}_{\mathrm{m}}{ }^{\mathrm{a}}$ gives equivalent path on $\mathfrak{D}_{\mathrm{m}}{ }^{\mathrm{b}}$ and vice versa. The equivalence of paths is determined by equality of path lengths.

Theorem 3: For any certain $m$, different $\mathscr{D}_{\mathrm{m}}$ s obey triangle law of addition in $X_{n} m<$ $n$, if addition impies successively traversing paths along the $\mathfrak{D}_{\mathrm{m}}$.

i.e. if points $x, y, z \in \mathrm{X}_{\mathrm{n}}$, then

$$
\mathscr{D}_{\mathrm{m}}(x y)+\mathscr{D}_{\mathrm{m}}(y x)=\mathscr{D}_{\mathrm{m}}(x z)
$$

Where, $\mathscr{D}_{\mathrm{m}}(i j)$ implies that the direction along the path going from $i$ to $j$ is realized as $\mathfrak{D}_{\mathrm{m}}$.

Proof: A $S_{n-1}$ having centre at point O accommodates many $S_{m-1}$ s for every $m<n$. The cross section of $S_{n-1}$ made by an $I_{m}$ is set of all points in the $I_{m}$ equidistant from O. Set of all points in $I_{m}$ equidistant from a point is nothing but a $S_{m-1}$. If the cross section contains O, then radius of $S_{m-1}$ is same as radius of the $S_{n-1}$. Otherwise $S_{m-1}$ has smaller radius, and has 
centre at projection of $\mathrm{O}$ on the $I_{m}$. Thus every cross section of $S_{n-1}$ made by an $I_{m}$ is a $S_{m-1}$. As $\mathscr{D}_{\mathrm{m}}$ is manifestation of path along $S_{m-1}$ (continuously varying $R_{m}$ ), the path along arbitrary cross section of $S_{n-1}$ made by a $I_{\mathrm{m}}$ leads to manifestation of $\mathscr{D}_{\mathrm{m}}$. Different cross sections of a $S_{n-1}$ made by different $I_{m}$ s in $X_{n}$ lead to manifestation of different $\mathscr{D}_{\mathrm{m}}$ s. $S_{\mathrm{n}-1}$ has infinitesimally $S_{m-1}$ structure in the cross section with $I_{m}$.

Consider left hand side of the equality as $\mathscr{D}_{\mathrm{m}}(x y)+\mathscr{D}_{\mathrm{m}}(y z)$. It implies that in $X_{n}, \mathscr{D}_{\mathrm{m}}$ along paths $x y \& y z$ exists. Thus existence of the bijections $R_{m}$ s from these paths in a frame are evident. According to the conjecture (which is used for defining $\mathscr{D} s$ ), all the points along path $x y$ should lie on a $S_{m-1}$ of radius r. Similarly all points along path $y z$ too lie on a $S_{m-1}$ of same radius $\mathrm{r}$ as it goes through common point $\mathrm{B}$. Hence points $x$ and $z$ lie on the same sphere of radius $\mathrm{r}$. As arbitrary cross section of $S_{n-1}$ made by an $I_{m}$ leads to manifestation of $\mathfrak{D}_{\mathrm{m}}$, for any two points $x \& z$ in the frame we can get a cross section to manifest $\mathscr{D}_{\mathrm{m}}$ along $x z$. Infinitely many cross sections going through two such points are possible. In order to be consistent with left hand side of $(8), \mathscr{D}_{\mathrm{m}}$ should be realized on a $S_{m-1}$ of same radius. This can be achieved if the $I_{m}$ goes through O. Thus we get a path on $S_{m-1}$ of radius $\mathrm{r}$ going from $x$ to $z$ all the points along which can be bijectively identified by the $R_{m}$ in the frame.

Conclusively, we have $\mathscr{D}_{\mathrm{m}}(x y)+\mathscr{D}_{\mathrm{m}}(y z)=\mathscr{D}_{\mathrm{m}}(x z)$ for any $x, y, z \in \mathrm{X}_{\mathrm{n}}$.

We learn that the cross section of $S_{n-1}$ made by the $I_{m}$ passing through centre of the $S_{n-}$ 1 is of importance over the cross sections by other $I_{m}$ s. Let us define such special cross sections those would be useful for analysis of $\mathscr{D}_{\mathrm{m}}$.

Definition 8: The cross section of $S_{n-1}$ made by an $I_{m}$ passing through centre of the $S_{n-1}$ is defined as an m-1 great sphere $\left(G_{m-1}\right)$ lying on the $S_{n-1}$ for $2 \leq m<n$.

$G_{m}$ is generalization of great circles. Infinitely many $G_{m}$ s are possible on $S_{n-1}$ due to various orientations of the $I_{m} \mathrm{~s}$ passing through $\mathrm{O}$. It is easy to check that radius of each $G_{m}$ is same as the $S_{n \geq m}$ on which it lies, as it has centre at $\mathrm{O}$ and contains points on $S_{n-1}$. Thus a $G_{m}$ goes through diametrically opposite points of $S_{n-1}$.

Theorem 4: All the independent $\mathcal{D}_{\mathrm{m}}$ s exist at each point on $S_{n-1}$ having equivalence relation with the independent $\mathcal{D}_{\mathrm{m}}$ s existing at all other points on the $S_{n-1}$.

Proof: $\mathscr{D}_{\mathrm{m}} \mathrm{s}$ are manifestations of paths along $S_{m-1} \mathrm{~s}$. An $I_{m}$ is determined by the $m+1$ points all of which don't belong to a single $I_{m-1}$. Thus arbitrary $m$ points on the $S_{n-1}$ that don't lie on a single $I_{m-1}$, along with the centre O determine an $I_{m}$ that can generate the $G_{m-1}$ for the $m$ points. If the points belong to single $I_{m-1}$, then $G_{m-2}$ is generated; there m-1 points suffice to specify $G_{m-2}$. Even lesser points suffice to specify a $G_{m-3}$. Only two points suffice to generate a $G_{1}$ i.e. great circle. Also, any $G_{m-1}$ has sufficient number of points to specify the $I_{m}$ containing it. This number is $m+1$. All the $m+1$ points can be on the $S_{n-1}$ or equivalently, $m$ points on the $S_{n-1}$ and remaining one at the centre O. Second set seems useful for analysis. 
Thus hereafter we will stick to proposition that the $m$ points on a $S_{n-1}$ and the centre O determine a $G_{m-1}$. It is also true that the $m$ points on $S_{n-1}$ alone can not determine a single $S_{m-1}$, they need one more point to specify a $S_{m-1}$.

Assume radius of the $S_{n-1}$ be r. Consider a $G_{m-1}$ specified by any $m$ points on $S_{n-1}$ and O. Path along this $G_{m-1}$ leads to realization of a $\mathscr{D}_{\mathrm{m}}$. To distinguish this specific $\mathfrak{D}_{\mathrm{m}}$ from other $\mathfrak{D}_{\mathrm{m}}$ on the $S_{n-1}$, label it $\mathfrak{D}_{\mathrm{m}} \mathrm{A}$. Also, $G_{m-1}$ A is label for the $G_{m-1}$ specified by the $m$ points.

Cross section of $S_{n-1}$ made by a $I_{m}$ parallel to $G_{m-1}{ }^{\text {A }}$ is $S_{m-1}$. As this $S_{m-1}$ doesn't go through $\mathrm{O}$, its radius is smaller than that of $G_{m-1} \mathrm{~A}$. Radius of such $S_{m-1}$ decreases as the parallel distance between the $I_{m}$ and $G_{m-1}{ }^{\mathrm{A}}$ increases. This is because, centre of such $S_{m-1}$ is at the gradually increasing distance $<\mathrm{r}$ from $\mathrm{O}$ (in Perpendiculal direction from $G_{m-1}{ }^{\mathrm{A}}$ ) and any point on $S_{m-1}$ is distance $\mathrm{r}$ from O. For a constant hypotenuse triangle, increase in one side implies the decrease in other. Let's denote the set of $S_{m-1} \mathrm{~S}$ parallel to $G_{m-1}{ }^{\mathrm{A}}$ by $\left\{S_{m-1}{ }^{\mathrm{A}}\right\}$. Gradual decrease in the radius with increase in the distance should lead to a zero radius. This is because, as $G_{m-1}{ }^{A}$ passes through $\mathrm{O}$, an $I_{m}$ can't intersect $S_{n-1}$ at a parallel distance more than $\mathrm{r}$. By definition of $S_{n-1}$, there should be only one point at distance $\mathrm{r}$ from O. Let's call this point as a pole with respect to $G_{m-1}$ A . There should be two poles with respect to $G_{m-1}{ }^{\mathrm{A}}$ determined by the parallel progressions in opposite directions. Both the poles are $2 \mathrm{r}$ apart from each other, thus they should be diametrically opposite. Both the poles exist in the intersections of $I_{m} \mathrm{~s}$ with $S_{n-1}$ that are parallel to $G_{m-1}$ A . Moreover, the poles contain the entire such intersections. A $I_{m}$ can be drawn passing through both the poles. Such $I_{m}$ is Perpendiculal to the $S_{m-1}$ s realized by the parallel progressions from $G_{m-1}$ A Specially, it is Perpendiculal to $G_{m-1}$. Thus, any $I_{m}$ going though both the poles should be Perpendiculal to $G_{m-1}{ }^{\mathrm{A}}$. The $I_{m}$ s going through the diametrically opposite poles will go through $\mathrm{O}$ and will generate a $G_{m-1}$. But $m+1$ points are needed to specify any $I_{m}$ or $G_{m-1}$. As only two points are there to generate this $G_{m-1}$, there is no certain such $G_{m-1}$ for $m>1$. Thus there are infinitely many such $G_{m-1}$ s specified by different points in addition to the poles. All such $G_{m-1}$ s are Perpendiculal to $G_{m-1}$ A . Let's denote set of all these $G_{m-1}$ s by $\left\{G_{m-1}{ }^{\mathrm{A}} \perp\right\}$. As $G_{m-1}$ is $S_{m-1}$, paths along them lead to manifestation of $\mathscr{D}_{\mathrm{m}}$. Thus all of $\left\{G_{m-1}{ }^{\mathrm{A}_{\perp}}\right\}$ lead to $\mathscr{D}_{\mathrm{ms}}$, let $\left\{\mathcal{D}_{\mathrm{m}}{ }^{\mathrm{A}_{\perp}}\right\}$ be their set. All $\left\{\mathscr{D}_{\mathrm{m}}{ }^{\mathrm{A}}{ }\right\}$ are Perpendiculal to $G_{m-1}{ }^{\mathrm{A}}$ and so to $\mathscr{D}_{\mathrm{m}}{ }^{\mathrm{A}}$, thus projections of them on $G_{m-1}{ }^{\mathrm{A}}$ would give zero path length along $\mathscr{D}_{\mathrm{m}}{ }^{\mathrm{A}}$. Hence all of $\left\{\mathscr{D}_{\mathrm{m}}{ }^{\mathrm{A}}{ }_{\perp}\right\}$ are independent directions with respect to $\mathscr{D}_{\mathrm{m}}{ }^{\mathrm{A}}$.

Each of $\left\{G_{m-1}{ }^{\mathrm{A} \perp}\right\}$ runs between both the poles with respect to $G_{m-1}{ }^{\mathrm{A}}$. Thus each of them should cross $G_{m-1}{ }^{\mathrm{A}}$. As each of $\left\{G_{m-1}{ }^{\mathrm{A} \perp}\right\}$ is perpendicular to $G_{m-1}{ }^{\mathrm{A}}$, no two of $\left\{G_{m-1}{ }^{\mathrm{A} \perp}\right\}$ will cross $G_{m-1}{ }^{\mathrm{A}}$ at same locations. This property can be used to distinguish different of $\left\{G_{m}\right.$ $\left.{ }^{\mathrm{A} \perp}\right\}$. We can use the metric induced by $R_{m}$ to distinguish different points on $G_{m-1}{ }^{\mathrm{A}}$. Suppose one of $\left\{G_{m-1}{ }^{\mathrm{A}} \perp\right.$ crosses $G_{m-1}{ }^{\mathrm{A}}$ at $x$, then it can be identified by $R_{m}(x)$ on $G_{m-1}{ }^{\mathrm{A}}$. Cross section of two $G_{m-1}$ S will be a curved $I_{m-2}$ (as it is the cross section of 3 geometrical entities: two $I_{m} \mathrm{~S}$ of the $G_{m-1}$ S and the $S_{n-1}$ ), and no $S_{m-1}$ can exist in $I_{m-2}$. Existence of the set $E_{x}$ on $S_{m-1}$ is essential for getting $R_{m}(x)$ according to (1). Due to inexistence of $S_{m-1}, E_{x}$ formed by $x$ on $G_{m-}$ ${ }_{1}^{\mathrm{A}}$ is empty. Thus $R_{m}(x)$ is zero. Though $R_{m}(x)$ is zero, for other cross sections on $G_{m-1}{ }^{\mathrm{A}}$, it provides a reference point $x$. If another of $\left\{G_{m-1}{ }^{\mathrm{A} \perp}\right\}$ crosses $G_{m-1}{ }^{\mathrm{A}}$ at $y$, then $x$ and $y$ existing 
on a $S_{m-1}$ facilitate the set $E_{x y}$ on its surface. Other reference points for configuration of $E_{x y}$ are provided by the cross section of $G_{m-1}^{\mathrm{A}}$ at $x$. Thus, $R_{m}(y)$ will be nonzero for all $y$. In this way, all cross sections of $G_{m-1}{ }^{\mathrm{A}}$ with each of $\left\{G_{m-1}{ }^{\mathrm{A} \perp}\right\}$ can be distinguished by the difference $R_{m}$ (i)- $R_{m}(x)$ or $R_{m}(x)-R_{m}(i)$. Each of $\left\{G_{m-1}{ }^{\mathrm{A} \perp}\right\}$ can cross $G_{m-1}{ }^{\mathrm{A}}$ at two diametrically opposite locations on $G_{m-1}{ }^{\mathrm{A}}$. Thus same element of $\left\{G_{m-1}{ }^{\mathrm{A} \perp}\right\}$ can have two different values of $R_{m}(x)$ $R_{m}(i)$. These two different values distinguish the two halves of the element of $\left\{G_{m-1}{ }^{\mathrm{A}_{\perp}}\right\}$ lying along different directions between the poles. Thus values of the metric $R_{m}(x)-R_{m}(i)$ configure two halves per element of $\left\{G_{m-1}{ }^{\mathrm{A}}{ }\right\}$ rather than the whole element. Both the halves disjointly cover the corresponding element. Such metric facilitates measurement of the projections of path lengths on $G_{m-1}{ }^{\mathrm{A}}$ as projected by $\left\{G_{m-1}{ }^{\mathrm{A}}\right\}$.

It isn't general to consider the $S_{m-1} \mathrm{~s}$ parallel to $G_{m-1} \mathrm{~s}^{\mathrm{A}}$ across only one direction. Multiple parallel such dimensions are possible on $S_{n-1}$ if $m<n$-1. If possible, the elements of $\left\{S_{m-1}{ }^{\mathrm{A}}\right\}$ also exist in a direction perpendicular to all the elements of $\left\{S_{m-1} \mathrm{~A}\right\}$ considered till now. Similar to as discussed above, we get the $G_{m-1}{ }^{\mathrm{A}_{\perp}} \mathrm{S}$ in this new direction. But all these $G_{m-}$ ${ }_{1}{ }^{\mathrm{A}_{\perp}}$ S are perpendicular to previously considered $S_{m-1}{ }^{\mathrm{A}} \mathrm{S}$, hence are perpendicular to all the $G_{m}$ ${ }_{1}^{\mathrm{A}_{\perp} \mathrm{S}}$ in the previously considered dimension. Let's denote the new set of $G_{m-1}{ }^{\mathrm{A}_{\perp}}$ by $\left\{G_{m}\right.$ $\left.1^{\mathrm{A} \perp \perp}\right\}$. All of $\left\{G_{m-1}{ }^{\mathrm{A} \perp \perp}\right\}$ are Perpendicular to all of $\left\{G_{m-1}{ }^{\mathrm{A}} \perp\right.$. If possible, there can be numerous such classes like $\left\{G_{m-1}{ }^{\mathrm{A} \perp \perp \perp}\right\},\left\{G_{m-1}{ }^{\mathrm{A} \perp \perp \perp \perp}\right\}$ etc, elements of all of them being mutually perpendicular. Also, all elements of $\left\{G_{m-1}{ }^{\mathrm{A}}\right\rfloor,\left\{G_{m-1}{ }^{\mathrm{A} \perp \perp}\right\},\left\{G_{m-1}{ }^{\mathrm{A} \perp \perp \perp}\right\}$ etc. are perpendicular to elements of $\left\{S_{m-1}{ }^{\mathrm{A}}\right\}$. It can be checked that one element from each of $\left\{G_{m-}\right.$ $\left.1^{\mathrm{A} \perp}\right\},\left\{G_{m-1}{ }^{\mathrm{A} \perp \perp}\right\},\left\{G_{m-1}{ }^{\mathrm{A} \perp \perp \perp}\right\}$ etc. and $\left\{S_{m-1}^{\mathrm{A}}\right\}$ exists at each point on the $S_{n-1}$ due to perfect symmetry of $S_{n-1}$. The $m$-dimensional ordered direction exists along all $G_{m-1} \mathrm{~s}$. Thus such directions exist along all elements of above sets. Let's denote set of the ordered directions along $\left\{G_{m-1}{ }^{\mathrm{A} \perp \perp}\right\}$ by $\left\{\mathscr{D}_{\mathrm{m}}{ }^{\mathrm{A} \perp \perp}\right\}$ and $\left\{G_{m-1}{ }^{\mathrm{A}_{\perp \perp \perp}}\right\}$ by $\left\{\mathscr{D}_{\mathrm{m}}{ }^{\mathrm{A}_{\perp \perp \perp}}\right\}$ and so on. With similar reasoning that made $\left\{\mathscr{D}_{\mathrm{m}}{ }^{\mathrm{A} \perp}\right\}$ independent of $\mathscr{D}_{\mathrm{m}}^{\mathrm{A}},\left\{\mathscr{D}_{\mathrm{m}}{ }^{\mathrm{A} \perp \perp}\right\},\left\{\mathscr{D}_{\mathrm{m}}{ }^{\mathrm{A} \perp \perp \perp}\right\}$ etc. are also independent direction w.r.t. $\mathscr{D}_{\mathrm{m}}{ }^{\mathrm{A}}$. And also, $\mathscr{D}_{\mathrm{m}}{ }^{\mathrm{A}},\left\{\mathscr{D}_{\mathrm{m}}{ }^{\mathrm{A} \perp}\right\},\left\{\mathscr{D}_{\mathrm{m}}{ }^{\mathrm{A} \perp \perp}\right\},\left\{\mathscr{D}_{\mathrm{m}}{ }^{\mathrm{A} \perp \perp \perp}\right\}$ etc. are mutually independent directions.

The elements of $\left\{G_{m-1}{ }^{\mathrm{A}} \perp\right\}$ facilitate projections of a path from $G_{m-1}{ }^{\mathrm{A}}$ on $\left\{S_{m-1}{ }^{\mathrm{A}}\right\}$. Such projections preserve the path length due to the compensating radius of certain $S_{m-1}{ }^{\mathrm{A}}$. Thus from definition 7 , the $\mathscr{D}_{\mathrm{m}} \mathrm{s}$ along all of $\left\{S_{m-1}{ }^{\mathrm{A}}\right\}$ are equivalent to $\mathscr{D}_{\mathrm{m}}{ }^{\mathrm{A}}$. Alternatively, $\mathfrak{D}_{\mathrm{m}}{ }^{\mathrm{A}}$ exists along $G_{m-1}{ }^{\mathrm{A}}$ and all of $\left\{S_{m-1}{ }^{\mathrm{A}}\right\}$.

All of $\left\{\mathscr{D}_{\mathrm{m}}{ }^{{ }^{\perp}}\right\}$ are perpendicular to $\mathscr{D}_{\mathrm{m}}{ }^{\mathrm{A}}$, hence all of them can be induced to have a relation with respect to $\mathscr{D}_{\mathrm{m}}{ }^{\mathrm{A}}$. Reflexivity, symmetry and transitivity of such relation among all elements of $\left\{\mathscr{D}_{\mathrm{m}}{ }^{\mathrm{A}_{\perp}}\right\}$ are compatible with the fact that the paths along them get equally projected on each other by $\left\{S_{m-1}{ }^{\mathrm{A}}\right\}$. Thus all of them are interchangeably independent of $\mathfrak{D}_{\mathrm{m}}{ }^{\mathrm{A}}$, and share equivalence relation. Therefore $\left\{\mathscr{D}_{\mathrm{m}}{ }^{{ }^{\perp}}\right\}$ can be written as a single direction $\mathscr{D}_{\mathrm{m}}{ }^{{ }^{\mathrm{A}_{\perp}}}$. In similar way, all elements of $\left\{\mathscr{D}_{\mathrm{m}}{ }^{\mathrm{A} \perp \perp}\right\}$ are independent directions of $\mathscr{D}_{\mathrm{m}}{ }^{\mathrm{A}}$ and $\mathscr{D}_{\mathrm{m}}{ }^{{ }_{\perp}}$ sharing the equivalence relation. Thus they can be represented by single direction $\mathscr{D}_{\mathrm{m}}{ }^{{ }^{\perp} \perp}$. Similarly all of $\left\{\mathscr{D}_{\mathrm{m}}{ }^{\mathrm{A} \perp \perp \perp}\right\},\left\{\mathscr{D}_{\mathrm{m}}{ }^{\mathrm{A} \perp \perp \perp \perp}\right\}$ etc can be represented by $\mathscr{D}_{\mathrm{m}}{ }^{\mathrm{A} \perp \perp \perp}, \mathfrak{D}_{\mathrm{m}}{ }^{\mathrm{A} \perp \perp \perp \perp}$ etc. respectively. In summary, $\mathscr{D}_{\mathrm{m}}{ }^{\mathrm{A}}, \mathfrak{D}_{\mathrm{m}}{ }^{\mathrm{A} \perp}, \mathscr{D}_{\mathrm{m}}{ }^{\mathrm{A} \perp \perp}, \mathfrak{D}_{\mathrm{m}}{ }^{\mathrm{A} \perp \perp \perp}$ etc. are the only mutually independent $\mathfrak{D}_{\mathrm{m}}$ on $S_{n-1}$, which have existence at each point on $S_{n-1}$ due to elements of $\left\{S_{m-1}{ }^{\mathrm{A}}\right\},\left\{G_{m-1}{ }^{\mathrm{A}} \perp\right\},\left\{G_{m-1}{ }^{\mathrm{A} \perp \perp}\right\}$, 
$\left\{G_{m-1}{ }^{\mathrm{A} \perp \perp \perp}\right\}$ etc. Hence all the independent $\mathscr{D}_{\mathrm{m}}$ exist at each point on $S_{n-1}$ having equivalence relation with the independent $\mathscr{D}_{\mathrm{m}}$ existing at all other points on the $S_{n-1}$.

Choice of the $G_{m-1}{ }^{\mathrm{A}}$ in above discussion is arbitrary. Set of $\mathscr{D}_{\mathrm{m}}{ }^{\mathrm{A}}, \mathscr{D}_{\mathrm{m}}{ }^{\mathrm{A} \perp}, \mathfrak{D}_{\mathrm{m}}{ }^{\mathrm{A} \perp \perp}$, $\mathscr{D}_{\mathrm{m}}{ }^{\mathrm{A} \perp \perp \perp}$ etc depend on this choice. There would be symmetry about this choice on $S_{n-1}$, but for now we can consider this choice be part of the frame of $\mathfrak{D}_{\mathrm{m}} \mathrm{s}$. We can proceed towards next result.

Theorem 5: Algebraic structure exists for the $\mathfrak{D}_{\mathrm{m}} \mathrm{s}$ with path lengths on $S_{n-1}$, for certain $m$.

Last theorem proves that all the independent $\mathscr{D}_{\mathrm{m}} \mathrm{s}$ exist at each point on $S_{n-1} . \mathscr{D}_{\mathrm{m}}$ is realization of changing $R_{m}$ along certain $S_{m-1}$. This can be casted into realization of moving certain path length along the $S_{m-1}$ by introducing a doublet consisting of the $\mathscr{D}_{\mathrm{m}}$ and $R_{m}$. Consider a set

$\mathrm{P}=\left\{\boldsymbol{p}^{\mathrm{a}}=p^{\mathrm{a}} \otimes \mathcal{D}_{\mathrm{m}}{ }^{\mathrm{a}}: \mathscr{D}_{\mathrm{m}}{ }^{\mathrm{a}} \in\left\{\mathscr{D}_{\mathrm{m}}{ }^{\mathrm{A}}, \mathscr{D}_{\mathrm{m}}{ }^{\mathrm{A}_{\perp}}, \mathscr{D}_{\mathrm{m}}{ }^{\mathrm{A} \perp \perp}, \mathscr{D}_{\mathrm{m}}{ }^{\mathrm{A}_{\perp \perp \perp}}\right.\right.$ etc. $\left.\}, p^{\mathrm{a}} \in \mathbb{R}\right\}$

here real number $p^{\mathrm{a}}$ can be attributed to certain path length along the $\mathscr{D}_{\mathrm{m}}{ }^{\mathrm{a}}$. Then each $p^{\mathrm{a}}$ can be expressed in terms of $R_{m}$ along the $\mathcal{D}_{\mathrm{m}}{ }^{\mathrm{a}}$. According to theorem 4 , any $\mathcal{D}_{\mathrm{m}}{ }^{\mathrm{a}}$ exists at each point on $S_{n-1}$. And as $\mathscr{D}_{\mathrm{m}}{ }^{\mathrm{a}}$ is along a $S_{m-1}$, the real valued $R_{m}$ can be traversed along the $\mathcal{D}_{\mathrm{m}}{ }^{\mathrm{a}}$ at each point on $S_{n-1}$. Thus existence of $\boldsymbol{p}^{\mathrm{a}}$ at $x$ can be attributed to traversing $R_{m}$ of magnitude $p^{\mathrm{a}}$ along the $\mathscr{D}_{\mathrm{m}}{ }^{\mathrm{a}}$ from $x$.

For $\boldsymbol{p}^{\mathbf{a}}$ and $\boldsymbol{p}^{\mathbf{b}} \in \mathrm{P}$, we can consider an operation of successively following the magnitudes and directions. That is if ' + ' denotes this operation, then

$$
p^{\mathrm{a}}+\boldsymbol{p}^{\mathrm{b}} \rightarrow \text { traverse } R_{m} \text { of } p^{\mathrm{a}} \text { along } \mathfrak{D}_{\mathrm{m}}{ }^{\mathrm{a}} \text {, then traverse } R_{m} \text { of } p^{\mathrm{b}} \text { along } \mathfrak{D}_{\mathrm{m}}{ }^{\mathrm{b}} \text { on } S_{n-1} \text {. }
$$

We can call this operation is addition. Further, we can define another operation (.) for any $c \in \mathbb{R}$ as

$c \cdot p^{\mathrm{a}} \rightarrow$ traverse $R_{m}$ of $p^{\mathrm{a}}$ multiplied by $c$ along $\mathscr{D}_{\mathrm{m}}{ }^{\mathrm{a}}$ on $S_{n-1}$.

This operation can be called as scaling. Note that both the operations are possible at any point on $S_{n-1}$ due to existence of all $\mathscr{D}_{\mathrm{m}}{ }^{\mathrm{a}} \mathrm{s}$ of (9) at all the points. As these operations are possible across $S_{n-1}$ along with existence of all $\boldsymbol{p} \in \mathrm{P}$ there, it is the algebraic structure existing for the $\mathscr{D}_{\mathrm{m}}$ with path lengths on $S_{n-1}$.

Due to above algebraic structures, independent $\mathscr{D}_{\mathrm{m}}$ can be combined to generate dependent $\mathscr{D}_{\mathrm{m}}$. Hence all the $\mathfrak{D}_{\mathrm{m}}$ spanning the $S_{n-1}$ can configured in terms of $\mathfrak{D}_{\mathrm{m}}{ }^{\mathrm{A}}, \mathfrak{D}_{\mathrm{m}}{ }^{\mathrm{A}}{ }$, 
$\mathscr{D}_{\mathrm{m}}{ }^{\mathrm{A} \perp \perp}, \mathscr{D}_{\mathrm{m}}{ }^{\mathrm{A} \perp \perp \perp}$ etc. of the frame. Thus the $\mathscr{D}_{\mathrm{m}} \mathrm{s}$ along $S_{m-1 \mathrm{~s}}$ inclined to $G_{m-1}{ }^{\mathrm{A}}$ also get configured algebraically. Due to existence of all $\mathscr{D}_{\mathrm{m}}{ }^{\mathrm{a}} \mathrm{s}$ of (9) at all the points along with the addition and scaling, the algebraic structure exists for all the $\mathfrak{D}_{\mathrm{m} S}$.

Path along any $\mathfrak{D}_{\mathrm{m}}$ existing on a $S_{m-1}$ of different radius can be projected on the $S_{m-1}$ of radius $\mathrm{r}$ by radial projections. Radial projections bijectively map each point between the spheres of different radii. Therefore the paths remain equivaleent and of equal lengths due to the same proportion of the radii and the Lebessgue measures on the surfaces. Thus paths along the $\mathfrak{D}_{\mathrm{m}} \mathrm{S}$ existing on 'coplanar' concentric $S_{m-1} \mathrm{~S}$ are equivalent. This makes such $\mathfrak{D}_{\mathrm{m}} \mathrm{S}$ equivalent according to definition 7 . Hence the algebra of $\mathscr{D}_{\mathrm{m}} \mathrm{s}$ spans whole $X_{n}$ rather than mere $S_{n-1}$.

Theorem 6: For a certain $m$, set of the $\mathfrak{D}_{\mathrm{m}} s$ with consideration of specific path length forms vector space.

Proof: Consider a set $\mathrm{V}_{\mathrm{m}}$ of all the $\mathscr{D}_{\mathrm{m}} \mathrm{s}$ in a frame on $X_{n}$ having associated with specific path length on the $S_{m-1}$ s.

$$
\text { i.e. } \mathrm{V}_{\mathrm{m}}=\left\{\boldsymbol{v}^{\mathrm{a}}=v \otimes \mathscr{D}_{\mathrm{m}}{ }^{\mathrm{a}}: v \in \mathbb{R} \text {, and } \mathscr{D}_{\mathrm{m}}{ }^{\mathrm{a}} \in\left\{\mathscr{D}_{\mathrm{m}}\right\}\right\}
$$

Elements of $\mathrm{V}_{\mathrm{m}}$ are $m$-dimensional ordered directions $\mathscr{D}_{\mathrm{m}}{ }^{\mathrm{a}} \mathrm{s}$ having certain path length $v$. By definition 2 , certain $\mathscr{D}_{\mathrm{m}}$ is realization of varying $R_{m}$. Furthermore, $R_{m}$ results to metric along a $\mathscr{D}_{\mathrm{m}}$ by lemma 3 . Thus the path length can be quantified in terms of $R_{m}$ of path extremities in the frame. Hence the elements of $\mathrm{V}_{\mathrm{m}}$ can be considered as a direct product of an ordered direction and the path length along it. Many $\mathscr{D}_{\mathrm{m}}$ are possible depending on number $n$ of dimensions of the configuration space $X_{n}$ in which the set $\mathrm{V}_{\mathrm{m}}$ is considered (as stated by corollary 2.1). Algebraic structure exists for $\mathscr{D}_{\mathrm{ms}}$ with path lengths as per the theorem 5. Thus we can explore addition and scaling among elements of $\mathrm{V}_{\mathrm{m}}$.

The product in (10) obeys rules of multiplication, thus $-v^{\mathrm{a}}$ implies that either $v$ or $\mathfrak{D}_{\mathrm{m}}{ }^{\mathrm{a}}$ has negative sign when compared to $\boldsymbol{v}^{\mathrm{a}}$. If $\mathscr{D}_{\mathrm{m}}$ a is realization of increasing $R_{m}$ on a path, then the $-\mathscr{D}_{\mathrm{m}}{ }^{\mathrm{a}}$ is manifestation of decreasing $R_{m}$ on same path. If a point object traverses path of length $v$ along $\mathscr{D}_{\mathrm{m}}{ }^{\mathrm{a}}$, then further traversing same $v$ along $-\mathscr{D}_{\mathrm{m}}{ }^{\text {a }}$ (or equivalently going $-v$ along $\mathcal{D}_{\mathrm{m}}{ }^{\mathrm{a}}$ ) will bring it to the initial point. Thus $\boldsymbol{v}^{\mathbf{a}} \&-\boldsymbol{v}^{\mathbf{a}}$ are inverses of each other under addition.

Denote elements of $\mathrm{V}_{\mathrm{m}}$ having either $v=0$ or absence of $\mathscr{D}_{\mathrm{m}}$ by $\mathbf{0}$. Then addition of $\mathbf{0}$ to a $\boldsymbol{v}^{\mathbf{a}}$ implies no variation in $\boldsymbol{v}^{\mathbf{a}}$. Also, addition of $\boldsymbol{v}^{\mathbf{a}}$ to $\mathbf{0}$ implies net $\boldsymbol{v}^{\mathbf{a}}$. Thus for any $\boldsymbol{v}^{\mathbf{a}} \in \mathrm{V}_{\mathrm{m}}$ we have $\boldsymbol{v}^{\mathbf{a}}+\mathbf{0}=\mathbf{0}+\boldsymbol{v}^{\mathbf{a}}=\boldsymbol{v}^{\mathbf{a}}$ i.e. $\mathbf{0}$ is identity element of $\mathrm{V}_{\mathrm{m}}$ under addition.

The $S_{m-1 \mathrm{~S}}$ are obtained as arbitrary cross sections of higher sphere $S_{i>\mathrm{m}}$ made by $I_{m} \mathrm{~s}$. As a $S_{m-1}$ represents the $I_{m}$ in which it exists, two $S_{m-1}$ s are perpendiculal or inclined only if corresponding $I_{m} \mathrm{~s}$ are so. Therefore such spheres can be adopted to facilitate projections of $\boldsymbol{v}^{\mathbf{a}}$ $\in \mathrm{V}_{\mathrm{m}}$ at desired points in $S_{n-1}$ thereby in $X_{n}$. Discussion in proof of the theorem 4 suggests existence of a unique $G_{m-1}$ passing through a point and perpendicular to a $S_{m-1}$. Thus 
projection of a $\boldsymbol{v}^{\mathbf{a}}$ on every other $\boldsymbol{v}^{\mathbf{b}}$ is defined due to existence of unique $I_{m}$ perpendicular to the $\mathscr{D}_{\mathrm{m}}{ }^{\mathrm{b}}$ (i.e. to $\boldsymbol{v}^{\mathbf{b}}$ ) and going through the extremity of $\boldsymbol{v}^{\mathbf{a}}$. A perpendicular $G_{m-1}$ would pass through the $G_{m-1}$ (e.g. along which $\boldsymbol{v}^{\mathbf{b}}$ lies) at two diametrically opposite points. In order to avoid degeneracy of the projection, the projection should be considered which doesn't go through any pole while projecting. Thus any point gets projected to a single point on the $G_{m-1}$. In this way, projection of a $\boldsymbol{v}^{\mathbf{a}}$ on every other $\boldsymbol{v}^{\mathbf{b}}$ is defined.

From theorem 5, algebraic structure of addition and scaling exists for $\mathfrak{D}_{\mathrm{m}} \mathrm{s}$ with path lengths. Thus the same exists on $\mathrm{V}_{\mathrm{m}}$. Due to this, any $\boldsymbol{v}^{\mathbf{a}}$ is equivalent to corresponding addition of the path lengths along the independent $\mathscr{D}_{\mathrm{m}} \mathrm{s}$. All the independent $\mathscr{D}_{\mathrm{m}} \mathrm{s}$ at the extremities of path lengths on each of independent $\mathscr{D}_{\mathrm{m}}$ form a closed parallelogram (or its higher dimensional analogue); where parallel coplanar sides represent same element. Then a diametrically opposite point of the parallelogram can be reached by the addition in variety of order of the elements. Thus order of addition doesn't matter for elements of $\mathrm{V}_{\mathrm{m}}$ existing along independent $\mathfrak{D}_{\mathrm{ms}}$.

Consider a point $o$ on the $S_{n-1}$ relative to which path lengths $v$ of all $v^{\text {a }}$ are defined. This is possible because all $\mathscr{D}_{\mathrm{m}}{ }^{\mathrm{a}} \mathrm{s}$ can be projected near $o$, and $R_{m}$ results to same metric along each of them. Let points $x$ and $y$ are described by $\boldsymbol{v}_{x}$ and $\boldsymbol{v}_{y} \in \mathrm{V}_{\mathrm{m}}$ respectively in this frame i.e. there is equivalence $o x \equiv \boldsymbol{v}_{x}=v_{x} \otimes \mathscr{D}_{\mathrm{m}}{ }^{\mathrm{a}}$, and $o y \equiv \boldsymbol{v}_{\boldsymbol{y}}=v_{y} \otimes \mathfrak{D}_{\mathrm{m}}{ }^{\mathrm{b}}$. Both $\boldsymbol{v}_{x}$ and $\boldsymbol{v}_{\boldsymbol{y}}$ can be resolved along the independent $\mathfrak{D}_{\mathrm{m}}{ }^{\mathrm{a}} \mathrm{s}$. Order doesn't matter for addition of the resolved elements along independent $\mathscr{D}_{\mathrm{m}}{ }^{\mathrm{a}} \mathrm{s}$. Also the order doesn't matter for addition of real numbers. Thus $\boldsymbol{v}_{x}+\boldsymbol{v}_{\boldsymbol{y}}$ should take to same point, as $\boldsymbol{v}_{\boldsymbol{y}}+\boldsymbol{v}_{x}$ should i.e. $\boldsymbol{v}_{x}+\boldsymbol{v}_{\boldsymbol{y}}=\boldsymbol{v}_{\boldsymbol{y}}+\boldsymbol{v}_{x}$. This is commutativity under addition of elements of $\mathrm{V}_{\mathrm{m}}$.

Theorems 4 and 5 imply equivalence of all elements of $\mathrm{V}_{\mathrm{m}}$ at all points on the $S_{n-1}$. Hence addition of the elements is associative under addition.

Elements of $\mathrm{V}_{\mathrm{m}}$ are direct products of real numbers and directions, and the algebraic structure of scaling exists. Thus $\mathrm{V}_{\mathrm{m}}$ holds the characteristics of scalar multiplication: compatibility of scalar multiplication with field multiplication, identity element of scalar multiplication, distributivity of scalar multiplication with respect to addition, distributivity of scalar multiplication with respect to field addition.

From above, all the axioms for a set to be vector space are satisfied and we can conclude that $\mathrm{V}_{\mathrm{m}}$ is a vector space.

Any point $x$ in the $X_{n}$ can be identified with an element $v_{x}$ of $\mathrm{V}_{\mathrm{m}}$ in certain frame.

There several $\mathscr{D}_{\mathrm{m}}$ are possible depending on dimensionality $n$ of the space. Different vector spaces $\mathrm{V}_{\mathrm{m}} \mathrm{s}$ having different value of $m$ lead to different realizations of the vector elements. For instance, elements of $V_{2}$ have angular direction while those of $V_{3}$ have direction enough to span 2-sphere. Elements of $V_{1}$ have rectilinear direction, though they 
don't exist on $S_{n-1}$ and their framework is general. Dimensionality of the configured elements of vector spaces is inherently intrinsic due to directions $\mathscr{D}_{\mathrm{m}}$ in their definition. Thus we can explicitly define the geometric vectors based on the Euclidean dimensions spanned by single element in the configuration space.

Definition 9: The m-dimensional vector is defined as element of $\mathrm{V}_{\mathrm{m}}$ having direction along a $\mathfrak{D}_{\mathrm{m}}$.

According to Theorem 2, in $n$-dimensional space we manifest $n$ types of ordered directions. Hence in $n$-dimensional space we have n-types of vectors viz. $m$-dimensional vectors with $m \leq n \in \mathbb{N}$. Also according to Theorem $1, R_{m}$ indicates direction along the $I_{m}$. There exists only one $I_{n}$, hence only one $\mathscr{D}_{\mathrm{n}}$ in $X_{n}$. Therefore $\mathrm{V}_{\mathrm{n}}$ has only one direction for all elements. Thus $\mathrm{V}_{\mathrm{n}}$ is not much useful for analysis in $X_{n}$ as the $\mathrm{V}_{\mathrm{m}}$ are.

We identify the scalar number field $v$ in definition (10) with range of $R_{m}$ along $\mathscr{D}_{\mathrm{m}}{ }^{\mathrm{a}}$ in same frame. For utilization of $\mathrm{V}_{\mathrm{ms}}$ for analysis on $X_{n}$, every $x \in X_{n}$ should be identified with single element of $\mathrm{V}_{\mathrm{m}}$. We have a simple scheme to do so. Any $x \in X_{n}$ can be considered on a path defined by single $\mathscr{D}_{\mathrm{m}}$. For this, we need the $m$ fixed points on the $S_{n-1}$ for quantification of $R_{m} \mathrm{~s}$. Then any point $x$ will lie along specific $\mathcal{D}_{\mathrm{m}}$ specified by cross section of corresponding $I_{m}$ with the $S_{n-1}$. The $m$ reference points fixed by the frame and $x$ define the cross sectional $I_{m}$. In this way specific direction $\mathscr{D}_{\mathrm{m}}$ a for every $x$ is identified. The scalar value corresponding to $x$ can be identified with path length given by $R_{m}$ of the $x$ from the reference point on the $S_{m-1}$. As $R_{m}$ is bijection upto period on the $\mathcal{D}_{\mathrm{m}}$, no two points have same path length in same direction $\left(\mathscr{D}_{\mathrm{m}}{ }^{\mathrm{a}}\right)$. Hence in order to identify points in $X_{n}$ with elements of $\mathrm{V}_{\mathrm{m}}$, we should use the mapping $v: X_{n} \rightarrow \mathrm{V}_{\mathrm{m}}$ given by

$$
\boldsymbol{v}(x)=R_{m}(x) \otimes \mathscr{D}_{\mathrm{m}}^{\mathrm{a}}(x)
$$

Where $R_{m}(x)$ is the path length of $x$ along the $\mathscr{D}_{\mathrm{m}}{ }^{\mathrm{a}}$ w.r.t. the reference points of the frame. Thus a vector space is direct product of the directions $\mathscr{D}_{\mathrm{m}}{ }^{\mathrm{a}} \mathrm{S}$ and range of $R_{m}$ for points along corresponding $\mathfrak{D}_{\mathrm{m}}{ }^{\mathrm{a}} \mathrm{s}$ in the configuration space.

Theorem 7: In $X_{n}, \mathrm{~V}_{\mathrm{m}} s \quad m \leq n \in \mathbb{N}$ are Banach spaces having the metric topology.

Proof: Theorem 6 concludes existence of vector space $\mathrm{V}_{\mathrm{m}}$ in $X_{n}$ and definition 2 defines it for all $m \leq n \in \mathbb{N}$. Elements of $\mathrm{V}_{\mathrm{m}}$ can be configured in $X_{n}$ by (11). Further, the configuration (11) facilitates a possible mapping $|v|: V_{m} \rightarrow \mathbb{R}$ for every element of $V_{m}$ as $|\boldsymbol{v}(x)|=R_{m}(x)$. In $X_{n}$, it is the $R_{m}(x)$ along $\mathscr{D}_{\mathrm{m}}{ }^{\mathrm{a}}$.

As $R_{m}$ is a measure by lemma 2 , for any $v \in \mathrm{V}_{\mathrm{m}}$, always $|\boldsymbol{v}(x)|=R_{m}(x) \geq 0$ i.e. $|\boldsymbol{v}(x)|$ is non negative. Further, when $|\boldsymbol{v}(x)|=R_{m}(x)=0$, it means that the $L_{m-1}\left(E_{x}\right)$ concerned by the conjecture is zero. In such case, no separation of the point $x$ from the reference point occurs; thus no manifestation of any path by $x$ and hence absence of any $\mathscr{D}_{\mathrm{m}}$. Thus in such case the 
element $\boldsymbol{v}$ has no direction i.e. $\boldsymbol{v}(x)=\mathbf{0}$. In other words, $R_{m}(x)=0$ and absence of any $\mathfrak{D}_{\mathrm{m}}$ in (11) yields $\boldsymbol{v}(x)=\mathbf{0}$. Conclusively we get non degeneracy of $|\boldsymbol{v}(x)|$ i.e. $|\boldsymbol{v}(x)|=0 \leftrightarrow \boldsymbol{v}(x)=\mathbf{0}$. For a scalar $\lambda$, we have $|\lambda \boldsymbol{v}(x)|=\lambda R_{m}(x)=\lambda|\boldsymbol{v}(x)|$. This is scalar multiplicativity of $|\boldsymbol{v}|$.

Further, for any $x, y \in X_{n}, \boldsymbol{v}(x)+\boldsymbol{v}(y)=R_{m}(x) \otimes \mathcal{D}_{\mathrm{m}}{ }^{\mathrm{a}}+R_{m}(y) \otimes \mathscr{D}_{\mathrm{m}}{ }^{\mathrm{b}} \cdot|\boldsymbol{v}|$ yields path length along the $\mathscr{D}_{\mathrm{m}}{ }^{\mathrm{a}}$ specified by $\boldsymbol{v}$. Algebraic structure exists for the path lengths along $\mathfrak{D}_{\mathrm{m}} \mathrm{S}$ and $\boldsymbol{v}(x)$ and $\boldsymbol{v}(y)$ are elements of a vector space, hence $\boldsymbol{v}(x), \boldsymbol{v}(y)$ and $\boldsymbol{v}(x)+\boldsymbol{v}(y)$ form a triangle on surface of $S_{n-1}$. We have the inequality for the path lengths along the triangle i.e. $|\boldsymbol{v}(x)+\boldsymbol{v}(y)| \leq|\boldsymbol{v}(x)|+|\boldsymbol{v}(y)|$.

As $|v|$ has essential properties of non negativity, non degeneracy, multiplicativity and triangle inequality on $\mathrm{V}_{\mathrm{m}},|v|$ is norm on $\mathrm{V}_{\mathrm{m}}$. $R_{m}$ of each element makes $\mathrm{V}_{\mathrm{m}}$ a normed vector space. This norm induces a metric $\mathrm{d}(\boldsymbol{v}(x), \boldsymbol{v}(y))=|\boldsymbol{v}(x)-\boldsymbol{v}(y)|=|\boldsymbol{v}(y)-\boldsymbol{v}(x)|$ and makes $\mathrm{V}_{\mathrm{m}}$ a metric space. This induces the usual metric topology on $\mathrm{V}_{\mathrm{m}}$.

Consider a sequence $\left\{\boldsymbol{v}\left(x_{i}\right)\right\}=\boldsymbol{v}\left(x_{1}\right), \boldsymbol{v}\left(x_{2}\right), \boldsymbol{v}\left(x_{3}\right), \ldots$ of elements of $\mathrm{V}_{\mathrm{m}}$ for points $x_{i}$ in $X_{n}$. Then due to continuity of $X_{n}$, there exists some index $\mathrm{N}$ for every real $\mathrm{r}>0$ such that $\mathrm{d}\left(\boldsymbol{v}\left(x_{i}\right), \boldsymbol{v}\left(x_{j}\right)\right)<\mathrm{r}$. whenever $i$ and $j$ are greater than $\mathrm{N}$. Thus $\left\{\boldsymbol{v}\left(x_{i}\right)\right\}$ is Cauchy sequence, and $\mathrm{d}$ is complete metric. This suggests that $\mathrm{V}_{\mathrm{m}}$ with $|v|$ is complete normed space i.e. Banach space.

It is worth to note that the mapping (11) is surjective for the range being algebraic vector space, due to surjectivity of the norm $R_{m}$. However, it is bijective upto the period of $R_{m}$. Such period is given by (7) where $\mathrm{f}_{\mathrm{i}}=1$. Thus, an arbitrary algebraic vector space can be configured as $\mathrm{V}_{\mathrm{m}}$ in $X_{n}$ if maxima of the scalar field fall under limit of such period. For the general scalar field, the algebraic elements those are the period apart (in terms of the norm induced metric) get configured to the same element of $\mathrm{V}_{\mathrm{m}}$ configured in $X_{n}$. However, geometry in $X_{n}$ explicitly leads to $\mathrm{V}_{\mathrm{m}}$ as discovered in last theorems.

From this point, one can derive all the aspects of conventional vectors spaces for $\mathrm{V}_{\mathrm{m}}$. We can check that the unit path lengths in terms of $R_{m}$ along all the independent $\mathscr{D}_{\mathrm{m}}$ in $X_{n}$ form basis of $\mathrm{V}_{\mathrm{m}}$ for each $m$.

Theorem 8: If an entity exists as a vector quantity in n-dimensional space then it exists in all the $n$ types of vectors as elements of $\mathrm{V}_{\mathrm{m} s} m \leq n \in \mathbb{N}$; and induces same dynamics with all the types.

Proof: If an entity exists as a vector quantity in $n$-dimensional space, then it has magnitude and direction in $n$-dimensional space $X_{n}$. The magnitude can be expressed as the path length along the direction in $X_{n}$. Mathematics is needed to keep scope for the general direction, and not to confine the existence of only certain specific kind of direction. A path along most general direction in $X_{n}$ can be expressed as resultant of the paths along all the 
ordered directions. Thus the magnitude along general direction can be expressed as sum of all the magnitudes along ordered directions by introducing an algebraic structure. An algebraic structure is defined by successively traversing the paths along the directions in constant frame. Therefore the general existence of the entity can be expressed as resultant of its components (or versions) along the ordered directions in $X_{n}$. Therefore the entity should exist as the path length along each kind of ordered direction in $X_{n}$ in order to quantify any infinitesimal component of it. Total $n$ kinds of ordered directions exist in $X_{n}$ as $\mathscr{D}_{\mathrm{m}}, m$ ranging from 1 to $n \in \mathbb{N}$. By theorem 6, path length along $\mathscr{D}_{\mathrm{m}}$ is element of a vector space $\mathrm{V}_{\mathrm{m}}$. Hence the entity should exist in all the $n$ types of vectors as elements of corresponding $\mathrm{V}_{\mathrm{m}} \mathrm{s} m \leq n \in$ $\mathbb{N}$. Theorem 2 implies existence of mutually exclusive ordered directions in $X_{n}$. Therefore variation in point object is along any of the $n$ types of ordered directions independently. An infinitesimal variation results in change in magnitude of any one type of vector (along any $\mathfrak{D}_{\mathrm{m}} \mathrm{m} \leq \mathrm{n}$ ) and not of other.

Despite of possibility of existence of different versions of the vector quantity, an underlying structure in $X_{n}$ is needed to facilitate existence of a vector version by offering infinitesimally piecewise corresponding direction. Elements of $\mathrm{V}_{1}$ have rectilinear direction while those of $\mathrm{V}_{\mathrm{m}>1}$ have directions along corresponding $S_{m-1} \mathrm{~s}$. Thus elements of $\mathrm{V}_{\mathrm{m}>1}$ can exist on the corresponding spheres only and not in general $X_{n}$, while elements of $\mathrm{V}_{1}$ can exist in general parts of $X_{n}$. This is because $X_{n}$ is infinitesimally piecewise rectilinear.

Here we considered the notion of magnitude and direction for existence of vector quantity. For the abstract vector, we can directly configure it in any version of vector in $X_{n}$ as algebra of all the $\mathrm{V}_{\mathrm{m}}$ is identical. Difference between different the versions arises when we concern for the nature of direction i.e. when we consider the vector geometrically under the notion of magnitude and direction.

Elements of an abstract vector space can be interpreted in $X_{n}$. Conventionally they are interpreted to be straight line segments $\left(\mathscr{D}_{1}\right)$, while now we can interpret them to be segments along any of $\mathscr{D}_{\mathrm{m}} \mathrm{s}$. For the new interpretation, dimensionality $n$ of $X_{n} \& m$ of the $\mathscr{D}_{\mathrm{m}}$ is important. In same $X_{n}$, dimensionality of $\mathrm{V}_{\mathrm{m}}$ varies with $m$ due to limitation on number of mutually perpendicular $I_{m} \mathrm{~S}$.

Possible underlying structure to facilitate existence of $\mathrm{V}_{2}$ in $X_{n}$ is solid spheres or bound circles. Solid spheres facilitate existence of the vectors along $\mathfrak{D}_{2}$ such as angular velocity, angular momentum, torque etc. Typically these vectors are considered along rectilinear direction by assigning right hand thumb rule as the morphism. It is algebraically fine as all the kinds of $\mathrm{V}_{\mathrm{ms}}$ form same abstract vector spaces. But the difference arises in geometry. These vectors indicate their difference when studied under symmetries. The scientific community compensated this matter by making two classes of vectors as pure vector (or polar vector) and pseudovector (or axial vector). Pseudovector is always associated with the cross product of two pure vectors [2], The pseudovectors don't obey laws of 
symmetry e.g. reflection. Reflection of $\mathfrak{D}_{2}$ is different from that of $\mathfrak{D}_{1}$ in same plane. We have some insights for the vector for $m>1$.

The trivial case of the application of above mathematical framework is of our physical universe. Let us see it as the example.

\section{Case of the universe}

Our universe can be identified with a 4-dimensional general manifold. Out of the four dimensions, locally 3 are spatial and 1 is temporal. Such space having 3 spatial dimensions and a parameter of evolution will be written as 3+1-dimensional space. More precisely, the universe $U$ is globally 4-dimensional while locally it is $3+1$-dimensional. Theorem 7 implies that for $\mathrm{n}=4, \mathrm{~V}_{\mathrm{m}} \mathrm{m} \leq 4, \mathrm{~m} \in \mathbb{N}$ form topological Banach spaces i.e. there would exist 4 types of vectors as elements of $\mathrm{V}_{1}, \mathrm{~V}_{2}, \mathrm{~V}_{3}$ and $\mathrm{V}_{4}$. Elements of $\mathrm{V}_{2}, \mathrm{~V}_{3}$ and $\mathrm{V}_{4}$ can exist on spheres only. Thus such higher dimensional vectors can exist on $S_{3}$ or equivalently 4-ball. Pretending the existance of the 4-balls, we can assume existence of the higher dimensional vectors on them.

The 4-dimensional vectors i.e. elements of $\mathrm{V}_{4}$ are useless for analysis. This is because in $\mathrm{U}$, single $\mathfrak{D}_{4}$ exists i.e. $\mathrm{V}_{4}$ configured in $\mathrm{U}$ is 1-dimensional Banach space; 1-dimensional vector space has least analytical value since it can be considered as scalar space. If linearly independent directions of vectors exist, then the vectors are useful for analysis. In this sense in $U$ there are three types of analytical vectors viz. 1-dimensional, 2-dimensional \& 3dimensional (4-dimensional being dormant for vector analysis).

1-dimensional vectors are the conventional vectors having directions along straight lines. 2-dimensional vectors have directions along $S_{1}$. While 3-dimensional vectors are having directions along $S_{2}$. In the immediate subsection, we will glampse on the 2dimensional vectors.

The case study of our universe is presented here purposefully. A theory in physics to be proposed in [3] concerns the universe as the configuration space accommodating four types of vectors.

\subsection{Angular Vectors}

It is well accepted that the infinitesimal angular rotations can be represented as vectors [4]. Angle is measure of arc of circle in plane. And as every section of the sphere made by a plane is a circle, every infinitesimal curve on circle can be measured in terms of angle (i.e. $R_{2}$ ). In general $R_{m}$ is measure on a $S_{m-1}$, and every cross section of $I_{m} \&$ higher sphere is $S_{m-1}$. Thus the higher spheres have infinitesimally piecewise $\mathscr{D}_{\mathrm{m}}$ structure to accommodate $m$-dimensional vectors.

Definition 7: Elements of $\mathrm{V}_{1}$ having direction along $\mathfrak{D}_{1}$ are defined as rectilinear vectors. 
Definition 8: Elements of $\mathrm{V}_{2}$ having direction along $\mathfrak{D}_{2}$ are defined as angular vectors.

Frame of $m$ points is needed for configuration of $\mathrm{V}_{\mathrm{m}}$. For configuration of rectilinear vectors $(m=1)$ in the frame, the origin in form of one point is needed. For angular vectors $(m=2)$, origin in form of a ray giving the centre and a point on every radius sphere is needed. The angular magnitudes are to be measured with respect to this ray. In contrast to rectilinear version, the angular vectors can exist on higher spheres or 4-balls only.

Algebraic expressions for all types of vectors are same such as linear combination of components, identities of dot product and cross product etc. This is valid if the magnitude in terms of $R_{m}$ is considered for $m$-dimensional vectors. As discussed in proof of theorem 7 , trivial norms for vectors are $R_{m}$ s i.e. distance, angle and solid angle correspondingly. But comparison of different typed vector magnitudes may be done by fixing all the quantifications $\left(R_{m}\right)$ in terms of distances. For this, we can exploit the conjecture. Angle can be written as ratio of arc and radius.

Basis can be identified for the vector spaces, wherein an arbitrary vector can be expanded in terms of basis vectors. Suppose an angular vector $\boldsymbol{x}$ is written as

$$
x=\mathrm{M} a+\mathrm{N} b
$$

where, $\mathrm{M} \& \mathrm{~N}$ are quantified in angles $\left(R_{2}\right)$ and $\boldsymbol{a}$ and $\boldsymbol{b}$ are basis angular vectors in $\mathrm{X}_{3}$. Then same can be written as

$$
\boldsymbol{x}=\frac{M}{\mathrm{r}} \boldsymbol{a}+\frac{N}{\mathrm{r}} \boldsymbol{b}
$$

where, $M \& N$ are quantified in distances $\left(R_{1}\right)$ on sphere of radius $\mathrm{r}$. The resultant vector and its components form spherical triangle on the $S_{2}$. We have equality from spherical trigonometry [5] as

$$
\cos (x)=\cos (\mathrm{M}) \cos (\mathrm{N})+\sin (\mathrm{M}) \sin (\mathrm{N}) \cdot \cos v
$$

where $x, \mathrm{M}$ and $\mathrm{N}$ are sides of spherical triangle formed on a sphere. $v$ is angle opposite to side $x$. The basis similar to $\mathscr{D}_{\mathrm{m}}{ }^{\mathrm{A}} \& \mathcal{D}_{\mathrm{m}}{ }^{\mathrm{A} \perp}$ (as concerned in proof of theorem 4 ) is possible. Then spherical triangle formed by the resultant angular vector and its components is right angled, i.e. if $x$ is resultant of $M \& N$, then $v^{\prime}=\frac{\pi^{\mathrm{c}}}{2}$. Hence second term in RHS of (13) vanishes. Thus using (12) \& (13) we get magnitude of angular vector as

$$
|\boldsymbol{x}|=\arccos (\cos (\mathrm{M}) \cos (\mathrm{N}))
$$

Further, we obtain unit angular vector as 


$$
\boldsymbol{u}=\frac{\boldsymbol{x}}{|\boldsymbol{x}|}=\frac{\mathrm{M} \boldsymbol{a}+\mathrm{N} \boldsymbol{b}}{\arccos (\cos (\mathrm{M}) \cos (\mathrm{N}))}
$$

Let two angular vectors in spatial universe $\mathrm{X}_{3}, \boldsymbol{x}=\mathrm{M} \boldsymbol{a}+\mathrm{N} \boldsymbol{b}$ and $\boldsymbol{y}=\mathrm{M}^{\prime} \boldsymbol{a}+\mathrm{N}^{\prime} \boldsymbol{b}$, then we get magnitude of the vector obtained by their addition as

$$
|\boldsymbol{x}+\boldsymbol{y}|=\arccos \left[\cos \left(\mathrm{M}+\mathrm{M}^{\prime}\right) \cdot \cos \left(\mathrm{N}+\mathrm{N}^{\prime}\right)\right]
$$

It can be checked that the essential triangle inequality $|x+y| \leq|x|+|y|$ holds for angular vectors.

The scalar product of two vectors is obtained as product of their projections on each other. Using the spherical law of sine [6] and (13), we obtain the scalar product of $\boldsymbol{x}$ and $\boldsymbol{y}$ as product of their projections on each other as follow

$$
\boldsymbol{x} . \boldsymbol{y}=\arccos \left(\frac{\cos |\boldsymbol{x}|}{\cos (\arcsin (\sin |\boldsymbol{x}| \cdot \sin \theta))}\right) \cdot \arccos \left(\frac{\cos |\boldsymbol{y}|}{\cos (\arcsin (\sin |\boldsymbol{y}| \cdot \sin \theta)}\right)
$$

where $\theta$ is angle between $\boldsymbol{x}$ and $\boldsymbol{y}$ on the higher sphere of existence.

It is easy to check that the scalar product (17) is commutative and fulfils desired properties of scalar product such as $\boldsymbol{x} \cdot \boldsymbol{x}=|\boldsymbol{x}|^{2}$, and for basis units $\boldsymbol{a} \cdot \boldsymbol{a}=1, \boldsymbol{b} \cdot \boldsymbol{b}=1$ and $\boldsymbol{a} \cdot \boldsymbol{b}=$ $\boldsymbol{b} . \boldsymbol{a}=0$. Using these relations for basis vectors, the scalar product in terms of components can be obtained as

$$
\boldsymbol{x} . \boldsymbol{y}=\left(\mathrm{MM}^{\prime}\right)+\left(\mathrm{NN}^{\prime}\right)
$$

This expression of scalar product is same as the abstract expression in terms of basis.

Vector product of two angular vectors can be developed using crux of vector product i.e. combination of perpendicular component of the vector acting on magnitude of other. If a vector $\boldsymbol{x}$ acts on another vector $\boldsymbol{y}$, then by geometric definition of cross product we take magnitude of component of $\boldsymbol{x}$ that is perpendicular to $\boldsymbol{y}$ and multiply it by magnitude of $\boldsymbol{y}$. Formulae for spherical trigonometry in [6] assists the derivation. Then we get the magnitude of cross product as

$|x \times y|=\left|x^{\prime}\right| \cdot|y| \quad$ where $\left|x^{\prime}\right|$ magnitude of component of $x$ that is perpendicular to $y$.

By using equations for spherical triangles, we get

$$
|\boldsymbol{x} \times \boldsymbol{y}|=\arccos \left(\frac{\cos |\boldsymbol{x}|}{\cos \left[\arcsin \left(\sin |\boldsymbol{x}| \cdot \sin \left(\frac{\pi}{2}-\theta\right)\right)\right]}\right) \cdot|\boldsymbol{y}|
$$


Idea behind the vector product implies that the vector product of two vectors is perpendicular to both of them. This is possible only if the product has direction linearly independent to that of both. In the example, $\boldsymbol{x}$ and $\boldsymbol{y}$ are expressed in terms of basis $\boldsymbol{a}$ and $\boldsymbol{b}$. Hence the vector product should have direction linearly independent to $\boldsymbol{a}$ and $\boldsymbol{b}$. Let's denote the unit vector in the new direction by $l$; thus the vector product (19) has direction $l$. That is,

$$
\boldsymbol{x} \times \boldsymbol{y}=\left[\arccos \left(\frac{\cos |\boldsymbol{x}|}{\cos \left\{\arcsin \left(\sin |\boldsymbol{x}| \cdot \sin \left(\frac{\pi}{2}-\theta\right)\right)\right\}}\right) \cdot|\boldsymbol{y}|\right] \boldsymbol{l}
$$

Using (20) we obtain the properties of angular vector product as

$$
\boldsymbol{a} \times \boldsymbol{a}=0 \text { and } \boldsymbol{b} \times \boldsymbol{b}=0 \text { and }|\boldsymbol{a} \times \boldsymbol{b}|=|\boldsymbol{b} \times \boldsymbol{a}|=1
$$

also $\boldsymbol{a} \times \boldsymbol{b}=\boldsymbol{l}$ and $\boldsymbol{b} \times \boldsymbol{a}=-\boldsymbol{l}$

Using these properties, in terms of basis we obtain (equivalent to general expression)

$$
\boldsymbol{x} \times \boldsymbol{y}=\left(\mathrm{MN}^{\prime}-\mathrm{NM}^{\prime}\right) \boldsymbol{l}=-(\boldsymbol{y} \times \boldsymbol{x})
$$

We have revealed basic details about 2-dimensional vectors or angular vectors which are elements of $\mathrm{V}_{2}$. The formulary is consistent with that of $\mathrm{V}_{1}$. Thus one may generalize the scalar and vector products for higher dimensional vectors in terms of basis. The algebraic properties of different types of vectors are identical. If angular vectors are identified to be rectilinear vectors by appropriate morphism, then algebraically one can't reveal the fact.

If we consider the angular vectors instead of pseudovectors, all physical systems are invariant under all trivial symmetry operations including reflection.

\subsection{Sangular Vectors}

As discussed earlier, in 4-dimensional space the vector space $\mathrm{V}_{3}$ having elements as 3-dimensional vectors can be configured. Such vectors will exist on the 4-balls (or 3-spheres) existing in $\mathrm{U}$; and have directions along $\mathscr{D}_{3}$ and norm in terms of $R_{3}$ i.e. solid angle. This norm will induce metric in terms of solid angle for the sangular vectors.

Definition 9: Elements of $\mathrm{V}_{3}$ having direction along $\mathfrak{D}_{3}$ are defined as sangular vectors.

$\mathrm{U}$ being 4-dimensional, can be configured as sangularly 2-dimensional vector space. Continuous random change in positions of a point object on surface of $S_{2}$ leads to manifestation of a sangular vector.

For sangular vectors in the frame, two points on $S_{2}$ are needed referring to which area traced by a point on $S_{2}$ (i.e. $E$ in the conjecture) can be measured. Both ends of a diameter 
can be considered as the reference points in the frame, these two points and the object point form triangle on the sphere. Area of such triangle divided by square of radius of the sphere yields the solid angle i.e. norm of the sangular vector of the object point in the frame. For quantification of area on the spheres, any two reference points would work, but we concluded end points of a diameter because this makes symmetry for choice of frames on the spheres. Further the end points of a diameter means $S_{0}$, this may help for generalization for higher dimensional vectors.

Area of the spherical triangle formed by two reference points and one object point characterizes norm of the sangular vector of the object point. Area A of plane triangle is half of the product of base and height (b.h/2); and area $A$ of spherical triangle having same base $b$ and height $\mathrm{h}$ has different but comparable area due to spherical excess. We can write $A=$ g.b.h/2 where, $\mathrm{g}$ is the deviation (function) due to spherical area. We don't need to explore $\mathrm{g}$ here.

For any sangular vector, the base concerned is constant as out of the three points, two are always reference points (i.e. end point of a diameter). The spherical distance between ends of a diameter is $\pi$ r i.e. $\mathrm{b}=\pi \mathrm{r}$. Using this substitution, we get area of the spherical triangle formed by point $x$ as $A_{x}=\mathrm{g} \pi \mathrm{r} . \mathrm{h}_{x} / 2$, where location of $x$ characterizes $\mathrm{h}_{x}$. Using this in the conjecture we get

$$
R_{3}(x)=\frac{{\mathrm{g} \pi \mathrm{h}_{x}}_{2}}{2 \mathrm{r}}
$$

\subsection{Vectors in the universe}

In the universe $\mathrm{U}$, rectilinear, angular and sangular types of vectors should exist. An angular vector spans two Euclidean dimensions as $S_{1}$ exists in 2-dimensional space. Similarly a sangular vector spans three Euclidean dimensions. Let in a frame, the four rectilinear basis dimensions of $\mathrm{U}$ are $x_{1}, x_{2}, x_{3}$ and $x_{4}$; let $x_{1}$ be time dimension. Let the unit angular vectors in planes $x_{1} x_{2}, x_{1} x_{3}$ and $x_{1} x_{4}$ be basis for $\mathrm{V}_{2}$ in same frame. Note that any combination $x_{i} x_{j}$ with $i$ being same and $j$ varying over three others forms basis for angular vector space, and all basis sets are equivalent as should be related by linear transformations.

In 4-dimensional space, only two linearly independent sangular vectors can exist and a sangular vector spans three rectilinear (Euclidean) dimensions.

In the universe $\mathrm{U}$, there exist three versions of vectors viz. rectilinear, angular and sangular. According to theorem 8, any quantity like displacement, momentum etc. should come in four versions as long as there is underlying structure to facilitate infinitesimal piecewise directions. The formalism on a version (or for general vector) is to be followed for all the versions of vectors. This means if rate of change (w.r.t. a quantity) of a vector quantity $\mathbf{v}$ is defined as $\mathbf{u}$, then it holds for all versions of vector as long as the underlying structure of say 4-ball exists to facilitate existence of $\mathbf{v}$ and the quantity. Therefore if a quantity is conserved, then it should be conserved in all typed vectors. 
According to Theorem 8, a vector quantity should exist in all the versions of vectors in general. If it changes in $U$, then it must change locally i.e. the change must be manifested in spatially 3-dimensional space with time evolution. If the change happens in magnitude, then it is manifested as change in the $R_{m}$ along the ordered direction. But if a vector of fixed magnitude exists and can change via variation in direction only, then local geometry on $U$ is important. If two linearly independent vectors of a version exist locally, then change in the vector via change in direction is manifested due to there are many vector directions possible. Local space of manifestation is spatial 3-dimensional portion of $U$ with time evolution. As in 3-dimensional space at least two linearly independent vectors of rectilinear and angular versions can exist, change in them due to direction is straightforward. This isn't the case with sangular vectors as only one such vector spans whole 3-dimensional (spatial) space.

According to theory of relativity, $\mathrm{U}$ is globally 4-dimensional continuum while locally is 3+1-dimensional having Minkowskian geometry. Thus if there exists 4-ball in $\mathrm{U}$, then locally it is manifested as 3-ball with one dimension being evolution parameter. Two linearly independent sangular vectors can exist on 4-ball, but only one such on 3-ball. The 3ball is projection (or cross section) of 4-ball aligned with local spatial space of manifestation $\mathrm{U}_{\mathrm{s}}$. If change in a sangular vector direction happens, then the change must be perpendicular to $\mathrm{U}_{\mathrm{s}}$. If a vector changes direction (or rotates) perpendicular to a subspace, then its projection on (or component in) the subspace should change. If a path along $\mathfrak{D}_{\mathrm{m}}$ having specific path length is changed (rotated) perpendicular to the accommodating $I_{m}$, then path length along the projection of the path in the $I_{m}$ will be changed depending on the amount of change (rotation). Thus even the path length is generally constant, for the projection in the subspace- it changes. Thus in effect, in local portion of $U$, change in sangular vector is manifested as change in its magnitude on the 3-ball (even if its magnitude on 4-ball is constant).

\subsection{Comparison of Magnitudes of different typed vectors}

Three versions of vectors exist in the universe. For fruitful analysis, comparison between magnitudes of different typed vectors is must. All the $m$-dimensional vectors with $m$ $>1$ exist on the respective spheres or balls. Rectilinear vectors are fundamental vectors quantified in terms of $R_{1}$. The universe is infinitesimally piecewise rectilinear. All the comparison should be done with respect to magnitude of rectilinear vector.

Consider a rectilinear vector $\mathbf{v}_{\mathrm{R}}$ of norm $\left|\mathbf{v}_{\mathrm{R}}\right|$, it should exist along $\mathscr{D}_{1}$ i.e. straight line. But the same set of points with same measure $L_{1}$ (i.e. curved line segment of the length $\left|\mathbf{v}_{\mathrm{R}}\right|$ ) can exist on spheres. This leads to norm of an angular vector along $\mathfrak{D}_{2}$. Magnitude of an angular vector $\mathbf{v}_{\mathrm{A}}$ is given by $\left|\mathbf{v}_{\mathrm{A}}\right|=E / \mathrm{r}, E$ being $L_{l}$ of the open set formed by the path extremities. The comparison can be obtained by substituting $\left|\mathbf{v}_{\mathrm{R}}\right|$ for $E$ meaning that same set of points with same measure is used to construct both the vectors. Thus we get

$$
\left|\mathbf{v}_{\mathrm{A}}\right|=\frac{\left|\mathbf{v}_{\mathrm{R}}\right|}{\mathrm{r}}
$$


Relation (23) provides comparison of magnitudes of the angular and rectilinear vectors if same amount of geometric content (in terms of Lebesgue measure) is used to generate both the vectors.

Norm of sangular vector is given by difference in $R_{3}$ s of its extremities. Thus norm $\left|\mathbf{v}_{S}\right|$ of sangular vector $\mathbf{v}_{S}$ is ratio of area due to $\mathbf{v}_{S}$ on the sphere to square of the radius. It is as given in (22). There $h_{x}$ is curved length which can be regarded as magnitude of the corresponding rectilinear vector for comparison. In other words, for comparison purpose $R_{3}(\mathrm{x})$ in (22) is magnitude of a sangular vector $\mathbf{v}_{\mathrm{S}}$ while $\mathrm{h}_{\mathrm{x}}$ is magnitude of a corresponding rectilinear vector $\mathbf{v}_{\mathrm{R}}$ when equivalent geometric content constitutes both the vectors. It takes the form

$$
\left|\mathbf{v}_{\mathrm{S}}\right|=\frac{\mathrm{g} \pi\left|\mathbf{v}_{\mathrm{R}}\right|}{2 \mathrm{r}}
$$

This equality provides abstract comparison of magnitudes. Here $\mathrm{g}$ is general function and we haven't explored it. The relative magnitudes of the three types of vectors may be helpful in theory.

\section{Conclusion}

Vectors have ordered directions that are not needed to be rectilinear always. The paper provides generalization of conventional interpretation of vectors. It concludes that a type of ordered direction exists for every number of Euclidean dimension. Path lengths along such ordered directions satisfy axioms of the vectors, hence they can be considered as vectors. Thus every number of dimensions comes with a version of vector. Algebra of all the typed vectors is identical. Expressions in terms of basis or components for scalar product and vector product are identical. But different typed vectors differ in magnitude; an $n$ dimensional vector has magnitude in terms of $R_{\mathrm{n}}$. Elements of arbitrary algebraic vector space may be interpreted as of any geometrical version in corresponding geometrical (configuration) space. All versions of the vector form Banach spaces and have metric induced topologies.

In 4-dimensional Euclidean space, three types of vectors exist viz. rectilinear, angular and sangular. A gross comparison of their magnitudes is obtained as (23) \& (24). The versions of the vectors retain their directions infinitesimally i.e. it is meaningless to say that an angular (or sangular) direction is infinitesimally rectilinear. This makes the generalized vectors different from those through the differential geometry.

Acknowledgements: Author dedicates the work to Jawaharlal Nehru. 


\section{References}

[1] S. Li, Asian. J. Math. Stat. (2011), 4: 66-70.

[2] A I Borisenko, I E Tarapov, Vector and tensor analysis with applications, Reprint of 1979 Prentice-Hall, Courier Dover p. 125

[3] G. S. Biraris, A theory with consolidation: Linking everything to explain everything, Results in Physics 7 (2017) 1650-1673

[4] R. Resnick, D Halliday, K K rane Physics Volume 1, John Willey \& sons, New Delhi (2004) p. 163

[5] B. O'Neill Elementary Differential Geometry, Academic Press. (2006) p. 441

[6] J. Sesiano Mathematics Across Cultures: the History of Non-western Mathematics, eds H Selin, U D'Ambrosio, Springer, Berlin (2000) p. 157 